\title{
NONMETRIZABLE TOPOLOGICAL DYNAMICS AND RAMSEY THEORY
}

\author{
VITALY BERGELSON AND NEIL HINDMAN
}

\begin{abstract}
Applying ideas from topological dynamics in compact metric spaces to the Stone-Cěch compactification of a discrete semigroup, several new proofs of old results and some new results in Ramsey Theory are obtained. In particular, two ultrafilter proofs of van der Waerden's Theorem are given. An ultrafilter approach to "central" sets (sets which are combinatorially rich) is developed. This enables us to show that for any partition of the positive integers one cell is both additively and multiplicatively central. Also, a fortuitous answer to a question of Ellis is obtained.
\end{abstract}

\section{INTRODUCTION}

Furstenberg and Weiss [13] proved a number of results in combinatorial partition theory (Ramsey Theory) using recurrence theorems in compact metric spaces. These methods were utilized for many additional impressive results (See [11].)

The current authors $[1,2,3$, and 17] have utilized this algebraic structure of $\beta N$, the Stone-Čech compactification of the set $N$ of positive integers, to obtain other results in Ramsey Theory. (These methods go back to the GalvinGlazer proof of the finite sum theorem.) When speaking about these results, we would very often be asked "Can you prove van der Waerden's Theorem in $\beta N$ ?." It was very annoying that the answer was "No," especially since van der Waerden's Theorem was needed for many of these results. The answer is changed by the current paper, in fact we present two very different proofs of van der Waerden's Theorem (or $2+1 / 2$ if we count Theorem 2.10 as $1 / 2$ ).

The work here is heavily influenced by work of Furstenberg and Katznelson on a density version of the Hales-Jewett Theorem [12]. We adapt arguments originally used in the context of enveloping semigroups and apply them to $\beta N$. There are three main advantages to these adaptations. The first is that the resulting arguments are actually simpler than the originals. The second is that

Received by the editors September 27, 1988. Presented to the Society, January 14, 1989 at Phoenix, Arizona.

1980 Mathematics Subject Classification (1985 Revision). Primary 54H20, 22A15; Secondary $05 \mathrm{~A} 17$.

Key words and phrases. Ramsey Theory, van der Waerden's Theorem, central sets.

The authors gratefully acknowledge partial support received from the National Science Foundation via grants DMS-85-05534 and DMS-85-20873 respectively. 
by departing from the metric situation we end up with results which are more widely applicable. The third is that, because $\beta N$ has two natural algebraic structures which interact, we end up with significant strengthenings of old results.

$\S 2$ is devoted to a proof of van der Waerden's Theorem based on that in [15] and an additional short proof of the three term version. We also answer an old question of Ellis in this section. $\S 3$ consists of an introduction to the main technique for the major results by way of another, and quite short, proof of van der Waerden's Theorem. In $\S 4$ we present the major results, generalizing results from [11] to a wide class of semigroups. In $\S 5$ we derive several corollaries, showing in particular that there is always a member of any finite partition of $N$ which is large in both a multiplicative and additive sense. We close in $\S 6$ with a proof that our notion of "central" agrees with that from [11] for any countable semigroup.

Recall that an ultrafilter $p$ on a set $S$ is a set of subsets of $S$ satisfying (i) $\varnothing \notin p$, (ii) $S \in p$, (iii) $A \in p$ and $A \subseteq B \subseteq S$ implies $B \in p$, (iv) $A \in p$ and $B \in p$ implies $A \cap B \in p$ and (v) if $r \in N$ and $S=A_{i} \cup A_{2} \cup \cdots \cup A_{r}$ then some $A_{i} \in p$. (In other words, an ultrafilter is a maximal filter.) Alternatively an ultrafilter $p$ may be identified with a finitely additive $\{0,1\}$-valued measure $\mu_{p}$ on $\mathscr{P}(S)$. Thus the statements $A \in p$ and $\mu_{p}(A)=1$ are synonymous.

Given a discrete set $S$ the points of the Stone-Cech compactification $\beta S$ of $S$ are taken as the ultrafilters on $S$. A point $s$ in $S$ is identified with the ultrafilter $\{A \subseteq S: s \in A\}$. Given $A \subseteq S$, let $\bar{A}=\{p \in \beta S: A \in p\}$. The set $\{\bar{A}: A \subseteq S\}$ forms a basis for the open sets (and a basis for the closed sets) of $\beta S$. If $(S,+)$ is a semigroup the operation can be extended to $\beta S$ making $(\beta S,+)$ a compact left-topological semigroup (that is, for each $p \in \beta S$ the function $\lambda_{p}$ defined by $\lambda_{p}(q)=p+q$ is continuous). This extension has the additional property that for each $x \in S$ the function $\rho_{x}$ defined by $\rho_{x}(q)=$ $q+x$ is continuous. The operation can be characterized by the fact that, given $A \subseteq S$ and $p, q \in \beta S, A \in p+q$ if and only if $\{x \in S: A-x \in p\} \in q$ where $A-x=\{y \in S: y+x \in A\}$. (If the operation is written "." we write $A / x=$ $\{y \in S: y \cdot x \in A\}$.) In any compact left-topological semigroup, idempotents exist [ 8 , Corollary 2.10$]$. See [20 or 21$]$ for an elementary derivation of these facts.

Observe that, given any $p \in \beta S, p+\beta S$ is a right ideal which is compact. (Indeed, $p+\beta S$ is the continuous image of $\beta S$ under $\lambda_{p}$.) Consequently one easily establishes via a routine Zorn's Lemma argument that any right ideal contains a minimal right ideal which is compact. We remark that not every right ideal is of the form $p+\beta S$. (For example, the smallest two-sided ideal of $\beta N$ is not closed [16, Corollary 3.10].) On the other hand, any minimal right ideal $R$ is representable in this form. Indeed if $q \in R$, then $q+\beta S \subseteq R+\beta S \subseteq R$ while $q+\beta S$ is a right ideal so $R=q+\beta S$.

We will need the following result. 
1.1 Lemma. Let $(S,+)$ be a discrete semigroup and let $p \in \beta S$. Then $p+\beta S$ is a minimal right ideal of $\beta S$ if and only if given any $A \subseteq S$, if there is some $x \in S$ with $A-x \in p$, then there is some finite $F \subseteq S$ such that for all $y \in S$, $\left(\bigcup_{x \in F} A-x\right)-y \in p$.

Proof. [19, Corollary 3.3].

In $\beta N$, the distributive laws fail badly. However, a special case does hold.

1.2 Lemma. Let $p, q \in \beta N$ and let $x \in N$. Then $(p+q) \cdot x=p \cdot x+q \cdot x$.

Proof. Since both $(p+q) \cdot x$ and $p \cdot x+q \cdot x$ are ultrafilters, it suffices to show $(p+q) \cdot x \subseteq p \cdot x+q \cdot x$. Let $A \in(p+q) \cdot x$. Then $A / x \in p+q$ so $B=\{y \in N:(A / x)-y \in p\} \in q$ and hence $B \cdot x \in q \cdot x$. We claim that $B \cdot x \subseteq\{y \in N: A-y \in p \cdot x\}$ (so that $A \in p \cdot x+q \cdot x$ ). To this end let $y \in B \cdot x$ and pick $z \in B$ with $y=z \cdot x$. Then $(A / x)-z \in p$ so $((A / x)-z) \cdot x \in p \cdot x$. Thus $(A / x) \cdot x-z \cdot x \in p \cdot x$. Since $(A / x) \cdot x \subseteq A$, we have $A-y \in p \cdot x$ as required.

The first infinite cardinal is $\omega=N \cup\{0\}$. Given a cardinal $\lambda$ we will sometimes write $[X]^{\lambda}=\{A \subseteq X:|A|=\lambda\}$ and $[X]^{<\lambda}=\{A \subseteq X:|A|<\lambda\}$.

\section{VAN DER WAERDEN'S THEOREM-}

APPROXIMATE SOLUTIONS TO EQUATIONS

The first proof of van der Waerden's Theorem which we present is an adaptation of the proof in $[15, \S 6.1]$. (This proof was itself adapted from [13].) The proof there uses metric space properties strongly and $\beta N$ is not metrizable (see $[14,14 \mathrm{~N}])$. We replace the notion of "closeness" by equivalence modulo a partition.

2.1 Definition. Let $\mathscr{E}$ be a finite partition of $N$ and let $p, q \in \beta N$. We write $p \approx q(\bmod \mathscr{E})$ if and only if for some $A \in \mathscr{E}, A \in p \cap q$ [i.e. $p, q \in \bar{A}$ where $\bar{A}=\{r \in \beta N: A \in r\}]$.

Also important to the proof in [15] is the notion of a minimal dynamical system. This notion is replaced by that of a minimal right ideal. The correspondence here is not accidental. $R$ is a minimal right ideal of $\beta N$ if and only if $R=p+\beta N$ for all $p \in R$. (If $p \in R$ and $R$ is minimal then $p+\beta N \subseteq R+\beta N \subseteq R$ and $p+\beta N$ is a right ideal. If $R=p+\beta N$ for all $p \in R$ and $T \subseteq R$ is a right ideal then given $p \in T, R=p+\beta N \subseteq$ $T+\beta N \subseteq T \subseteq R$.) Since a minimal right ideal $R$ is a compact left topological semigroup, it has an idempotent $p$, which then satisfies $R=p+\beta N$. Also $p+\beta N=\operatorname{cl}\{p+n: n \in N\}$ a forward orbit closure under the transformation $T(p)=p+1$. If $p+\beta N$ is a minimal right ideal, then $(p+\beta N, T)$ is a minimal dynamical system.

We shall utilize the notion of $p-\lim _{n \in N} x_{n}$ (introduced by Frolík in [10]).

2.2 Definition. Let $I$ be a set, $X$ a topological space, $\left\langle x_{\alpha}\right\rangle_{\alpha \in I}$ an indexed family in $X$ and $p$ an ultrafilter on $I$. We write $p-\lim _{\alpha \in I} x_{\alpha}=z$ if and only if 
$z \in X$ and whenever $U$ is a neighborhood of $z$ one has $\left\{\alpha \in I: x_{\alpha} \in U\right\} \in p$.

Observe that $p$ - $\lim _{\alpha \in I} x_{\alpha}$ must exist in a compact space and is unique in a Hausdorff space.

The following lemma is well known in the context of metric dynamical systems.

2.3 Lemma. Let $R$ be a minimal right ideal of $(\beta N,+)$ and let $\mathscr{E}$ be a finite partition of $N$. There exists $k \in N$ such that for any $q, r \in R$ there exists some $t \in\{1,2, \ldots, k\}$ with $q+t \approx r(\bmod \mathscr{E})$.

Proof. Suppose the assertion is wrong. Then for any $n$ there exists $q_{n}, r_{n} \in R$ such that $q_{n}+t \not \approx r_{n}(\bmod \mathscr{E})$ for all $t \in\{1,2, \ldots, n\}$. Let $p$ be any nonprincipal ultrafilter on $N$. Let $q=p-\lim _{n \in N} q_{n}$ and $r=p-\lim _{n \in N} r_{n}$.

Choose $A \in \mathscr{E}$ with $A \in r$. Then $\bar{A}$ is a neighborhood of $r$ and $r \in R=$ $\operatorname{cl}\{q+n: n \in N\}$ so there exists $t_{0} \in N$ with $q+t_{0} \in \bar{A}$. Now $p-\lim _{n \in N} q_{n}=q$ and $A-t_{0} \in q$ so $\left\{n \in N: A-t_{0} \in q_{n}\right\} \in p$. Likewise $p-\lim _{n \in N} r_{n}=r$ and $A \in r$ so $\left\{n \in N: A \in r_{n}\right\} \in p$. We may thus choose $n>t_{0}$ with $A-t_{0} \in q_{n}$ and $A \in r_{n}$. But then

$$
q_{n}+t_{0} \approx q+t_{0} \approx r \approx r_{n} \quad(\bmod \mathscr{E}),
$$

contradicting our supposition.

2.4 Lemma. Let $R$ be a minimal right ideal of $(\beta N,+)$, let $q \in R, n \in N$ and define $q-n=\{A-n: A \in q\}$. Then $q-n \in R$.

Proof. It is routine to verify that $q-n$ is an ultrafilter (using the fact that $q$ is nonprincipal). Now $q \in R=q+\beta N$ so pick $r \in \beta N$ such that $q=q+r$. One easily sees that $r \in \beta N \backslash N$ (since no congruence class $\bmod (m+1)$ could be in both $q$ and $q+m$ for $m \in N)$. Thus $r-n \in \beta N \backslash N$. Finally one routinely verifies that $(q+r)-n=q+(r-n)$ so that $q-n \in q+\beta N=R$.

The current proof in fact provides something stronger than van der Waerden's Theorem; one is allowed to choose the increment from any prescribed set of finite sums. (This is not new. It is derivable from the Hales-Jewett theorem and is explicitly in [11].) For $B \subseteq N$, we write $F S(B)=\left\{\sum F: F\right.$ is a finite nonempty subset of $B\}$. Given $d_{1}, d_{2} \in F S(B)$ we write $d_{1} \prec d_{2}$ if whenever $d_{1}=\sum F_{1}$ and $d_{2}=\sum F_{2}$ with $F_{1}, F_{2} \subseteq B$ one has $\max F_{1}<\min F_{2}$.

The statements defined below depend on the right ideal $R$ as well as on $l$. We suppress reference to $R$ since it will remain fixed.

2.5 Definition. Let $l \in N \cup\{0\}$ and let $R$ be a minimal right ideal of $(\beta N,+)$.

(a) $S(l)$ is the statement: "for each finite partition $\mathscr{E}$ of $N$ and each infinite $B \subseteq N$ there exist $q \in R$ and $d \in F S(B)$ such that for each $i \in$ $\{0,1, \ldots, l\}, q \approx q+\mathrm{id}(\bmod \mathscr{E}) . "$

(b) $T(l)$ is the statement: "for each finite partition $\mathscr{E}$ of $N$, each infinite $B \subseteq N$, each $s \in R$ and each $d_{0} \in F S(B)$ there exist $q \in R$ and $d \in F S(B)$ such that $d_{0} \prec d$ and for each $i \in\{1,2, \ldots, l\}, s \approx q+\mathrm{id}(\bmod \mathscr{E})$." 
The result we are after is that for all $l S(l)$ holds. This is accomplished by the next two lemmas. In these, an $\varepsilon-\delta$ argument has been replaced by a mechanism for producing appropriately finer partitions. The use of the letters $\mathscr{E}$ and $\mathscr{D}$ in these proofs is intended to be suggestive of this replacement.

2.6 Lemma. Let $l \in N$ and let $R$ be a minimal right ideal of $(\beta N,+)$,

$$
S(l-1) \Rightarrow T(l) \text {. }
$$

Proof. Assume $S(l-1)$. Let $\mathscr{E}, B, s$, and $d_{0}$ be given as in the definition of $T(l)$. Pick $k$ as guaranteed by Lemma 2.3 for $R$ and $\mathscr{E}$. Let $\mathscr{D}$ be the partition induced by $\{A-i: i \in\{0,1, \ldots, k\}$ and $A \in \mathscr{E}\}$. Let $B^{\prime}=\{x \in$ $\left.B: x>d_{0}\right\}$. Since $S(l-1)$ holds, pick $q^{\prime} \in R$ and $d \in F S\left(B^{\prime}\right)$ such that for each $i \in\{0,1, \ldots, l-1\}, q^{\prime} \approx q^{\prime}+\mathrm{id}(\bmod \mathscr{D})$. By the choice of $k$, pick $j \in\{1,2, \ldots, k\}$ such that $q^{\prime}+j \approx s(\bmod \mathscr{E})$. Let $q=\left(q^{\prime}-d\right)+j$.

Observe immediately that $d_{0} \prec d$. Now let $i \in\{1,2, \ldots, l\}$. We need to show that $s \approx q+\mathrm{id}(\bmod \mathscr{E})$. Now $i-1 \in\{0,1, \ldots, l-1\}$ so $q^{\prime} \approx$ $q^{\prime}+(i-1) d(\bmod \mathscr{D})$ so by the choice of $\mathscr{D} q^{\prime}+j \approx q^{\prime}+(i-1) d+j(\bmod \mathscr{E})$. That is $q^{\prime}+j \approx q+\mathrm{id}(\bmod \mathscr{E})$. Since $q^{\prime}+j \approx s(\bmod \mathscr{E})$ we have $s \approx q+\mathrm{id}$ $(\bmod \mathscr{E})$ as required.

2.7 Lemma. Let $l \in N$ and let $R$ be a minimal right ideal of $(\beta N,+)$.

$$
T(l) \Rightarrow S(l)
$$

Proof. Assume $T(l)$.

Given any finite partition $\mathscr{E}$ and any $d$ in $N$, let $\mathscr{D}(\mathscr{E}, d)$ be the partition induced by $\{A-\mathrm{id}: i \in\{0,1, \ldots, l\}$ and $A \in \mathscr{E}\}$.

Let a partition $\mathscr{E}=\mathscr{E}_{0}$ and infinite $B \subseteq N$ be given and let $n=\left|\mathscr{E}_{0}\right|$, the number of cells of $\mathscr{E}_{0}$. Pick $q_{0} \in R$ arbitrarily. Using $T(l)$ pick $q_{1} \in R$ and $d_{1} \in F S(B)$ such that for each $i \in\{1,2, \ldots, l\}, q_{0} \approx q_{1}+\mathrm{id}_{1}\left(\bmod \mathscr{E}_{0}\right)$. Let $\mathscr{E}_{1}=\mathscr{D}\left(\mathscr{E}_{0}, d_{1}\right)$. Again using $T(l)$ pick $q_{2} \in R$ and $d_{2} \in F S(B)$ with $d_{1} \prec d_{2}$ and so that for each $i \in\{1,2, \ldots, l\}, q_{1} \approx q_{2}+\mathrm{id}_{2}\left(\bmod \mathscr{E}_{1}\right)$.

Continuing in this way, we have for $i \in\{1,2, \ldots, l\}$ :

$$
\begin{gathered}
q_{0} \approx q_{1}+\mathrm{id}_{1}\left(\bmod \mathscr{E}_{0}\right) \\
q_{1} \approx q_{2}+\mathrm{id}_{2}\left(\bmod \mathscr{E}_{1}\right) \\
\vdots \\
q_{n} \approx q_{n+1}+\mathrm{id}_{n+1}\left(\bmod \mathscr{E}_{n}\right)
\end{gathered}
$$

where, for $j \in\{0,1, \ldots, n-1\}, \mathscr{E}_{j+1}=\mathscr{D}\left(\mathscr{E}_{j}, d_{j+1}\right)$.

Since $n=\left|\mathscr{E}_{0}\right|$, we pick by the pigeonhole principle some $t, m$ with $1 \leq t<$ $m \leq n+1$ so that $q_{t} \approx q_{m}\left(\bmod \mathscr{E}_{0}\right)$. Let $q=q_{m}$ and $d=d_{t+1}+d_{t+2}+\cdots+d_{m}$. Observe that since each $d_{j-1} \prec d_{j}$ we have $d \in F S(B)$. To complete the proof let $i \in\{1,2, \ldots, l\}$. We need to show that $q \approx q+\mathrm{id}\left(\bmod \mathscr{E}_{0}\right)$. 
First $q_{m-1} \approx q_{m}+\mathrm{id}_{m}\left(\bmod \mathscr{E}_{m-1}\right)$. (If it happens that $t=m-1$, we are done since then $q=q_{m} \approx q_{t}=q_{m-1} \approx q_{m}+\mathrm{id}_{m}=q+\mathrm{id}$.). Now $\mathscr{E}_{m-1}=\mathscr{D}\left(\mathscr{E}_{m-2}, d_{m-1}\right)$ so from $q_{m-1} \approx q_{m}+\mathrm{id}_{m}\left(\bmod \mathscr{E}_{m-1}\right)$ we conclude $q_{m-1}+\mathrm{id}_{m-1} \approx q_{m}+\mathrm{id}_{m}+\mathrm{id}_{m-1}\left(\bmod \mathscr{E}_{m-2}\right)$. Since also $q_{m-2} \approx q_{m-1}+\mathrm{id}_{m-1}$ $\left(\bmod \mathscr{C}_{m-2}\right)$ we have $q_{m-2} \approx q_{m}+i\left(d_{m}+d_{m-1}\right)\left(\bmod \mathscr{E}_{m-2}\right)$. Continuing in this way we eventually get $q_{t} \approx q_{m}+i\left(d_{m}+d_{m-1}+\cdots+d_{t+1}\right)\left(\bmod \mathscr{E}_{t}\right)$. Since $\mathscr{E}_{t}$ refines $\mathscr{E}_{0}$ we have $q=q_{m} \approx q_{t} \approx q_{m}+i\left(d_{m}+d_{m-1}+\cdots+d_{t+1}\right)=q+\mathrm{id}$, with all congruences $\bmod \mathscr{E}_{0}$.

2.8 Theorem. Let $R$ be a minimal right ideal of $(\beta N,+)$. For all $l \in$ $N \cup\{0\}, S(l)$ holds.

Proof. $S(0)$ holds trivially so apply Lemmas 2.6 and 2.7.

2.9 Corollary (van der Waerden). Let $l \in N$, let $B$ be an infinite subset of $N$ and let $\mathscr{E}$ be a finite partition of $N$. There exist $A \in \mathscr{E}, a \in N$, and $d \in F S(B)$ such that $\{a, a+d, \ldots, a+l d\} \subseteq A$.

Proof. Let $R$ be any minimal right ideal of $(\beta N,+)$ and pick $q \in R$ as guaranteed by $S(l)$. Pick $A \in \mathscr{E}$ such that $A \in q$. Then for each $i \in$ $\{0,1, \ldots, l\}, A-\mathrm{id} \in q$ so pick $a \in \bigcap_{i=0}^{l} A-\mathrm{id}$.

We now present an extremely short proof of the simplest nontrivial version of van der Waerden's Theorem. Unfortunately, as we shall see, this proof does not generalize in the obvious way to longer arithmetic progressions. Note that $2 p$ means $2 \cdot p$ in $(\beta N, \cdot)$ and $2 p$ is not in general equal to $p+p$.

2.10 Theorem. Let $p \in \beta N$ with $p=p+p$. Then for each $A \in p+2 p$, there exist $a, d \in N$ with $\{a, a+d, a+2 d\} \subset A$.

Proof. By Lemma 1.2, $2 \cdot p=p \cdot 2=(p+p) \cdot 2=p \cdot 2+p \cdot 2=2 p+2 p$. Also observe that, given $r \in \beta N$ and $B \subseteq N, B \in r+2 p$ if and only if $\{n \in N ; B-2 n \in r\} \in p$.

Let $A \in p+2 p$. Let $B=\{n: A-2 n \in p\}$ and $C=\{n: A-2 n \in p+2 p\}$. Since $A \in p+2 p, B \in p$ and since $A \in p+2 p=p+2 p+2 p, C \in p$. Pick $n \in B \cap C$. Let $D=\{d: A-2 n-d \in p\}$ and $E=\{d: A-2 n-2 d \in p\}$. Since $A-2 n \in p=p+p, D \in p$. Since $A-2 n \in p+2 p, E \in p$. Pick $d \in D \cap E$. Then $A-2 n \in p, A-2 n-d \in p$, and $A-2 n-2 d \in p$. Pick $b \in(A-2 n) \cap(A-2 n-d) \cap(A-2 n-2 d)$ and let $a=b+2 n$.

Note that, in the above proof, since $(A-2 n) \cap(A-2 n-d) \cap(A-2 n-2 d) \in$ $p=p+p$, we can choose infinite $G$ with $F S(G) \subseteq(A-2 n) \cap(A-2 n-d) \cap$ $(A-2 n-2 d)$. (Here $F S(G)=\left\{\sum H: H \subseteq G\right.$ and $H$ is finite and $\left.H \neq \varnothing\right\}$.) That is, the beginning of the arithmetic progression may be chosen as any one of $2 n+F S(G)$.

By virtue of Theorem 2.10, one can approximately solve the equation $r=$ $r+p=r+2 p$ in $\beta N \backslash N$, with $p$ an idempotent. (That is, given any finite partition $\mathscr{F}$ of $N$ one can find $q \in \beta N \backslash N$ and an idempotent $p$ with 
$q \approx q+p \approx q+2 p(\bmod \mathscr{F})$.) To see this, let $p$ be an idempotent. Given $\mathscr{F}$, pick $A \in \mathscr{F}$ with $A \in p+2 p$. Pick $n$ as in the proof of Theorem 2.10 and let $q=p+2 n$. Without much difficulty one can show that $A \in q \cap(q+p) \cap(q+2 p)$. (In general, if there exist $q, p$ such that $A \in \bigcap_{i=0}^{n-1}(q+i p)$ then $A$ contains a length $n$ arithmetic progression.)

If we could exactly solve the equation $q+p=q+2 p$, a proof similar to that of Theorem 2.10 shows that every member of $q+p+3 p$ contains a 4 term arithmetic progression. We show now that this is impossible. (The question of which equations are solvable in $\beta N$ also has some independent interest.)

We remark that Theorem 2.10 sheds some light on the question of where triples "live." We have no such, even partial, characterization related to quadruples.

2.11 Theorem. Let $q, p \in \beta N$. Then $q+p \neq q+2 p$.

Proof. For each $n \in N$, pick $a_{n} \in\left\{0,1,2, \ldots, 3^{n}-1\right\}$ such that $\{x \in$ $\left.N: x \equiv a_{n}\left(\bmod 3^{n}\right)\right\} \in q$ and let $g_{n}:\{0,1,2, \ldots, n-1\} \rightarrow\{0,1,2\}$ such that $a_{n}=\sum_{k=0}^{n-1} g_{n}(k) \cdot 3^{k}$. (Thus $g_{n}$ gives the ternary representation of $a_{n}$.) Note that if $m<n$, then since $\left\{x \in N: x \equiv a_{n}\left(\bmod 3^{n}\right)\right\} \cap\left\{x \in N: x \equiv a_{m}\right.$ $\left.\left(\bmod 3^{m}\right)\right\} \neq \varnothing$, we have $\left.g_{n}\right|_{m}=g_{m}$. Let $g=\bigcup_{n=1}^{\infty} g_{n}$. Given any $x \in N$ pick $h_{x}: \omega \rightarrow\{0,1,2\}$ so that $x=\sum_{k=0}^{\infty} h_{x}(k) \cdot 3^{k}$ (so that $h_{a_{n} l_{n}}=g_{n}$ ).

Given $x \in N$ if $h_{x}=g$ (which can happen at most once) let $l(x)=\infty$. Otherwise, let $l(x)=\min \left\{t \in \omega: h_{x}(t) \neq g(t)\right\}$. For $i \in\{0,1,2\}$ let $A_{i}=$ $\left\{x \in N: l(x)<\infty\right.$ and $\left.h_{x}(l(x))=i\right\}$ and let $A_{3}=\{x \in N: l(x)=\infty\}$. Suppose that $q+p=q+2 p$ and pick $i \in\{0,1,2,3\}$ such that $A_{i} \in q+p$. Then $\left\{x \in N: A_{i}-x \in q\right\} \in p$ and $\left\{x \in N: A_{i}-2 x \in q\right\} \in p$ so pick $x \in N$ with $A_{i}-x \in q$ and $A_{i}-2 x \in q$. Pick $m$ such that $x<3^{m}$ and pick $y \in\left\{y \in N: y \equiv a_{m}\left(\bmod 3^{m}\right)\right\} \cap A_{i}-x \cap A_{i}-2 n$. Let $t=\min \left\{k \in \omega: h_{x}(k) \neq\right.$ $0\}$ and note that $t<m$ and $t=\min \left\{k \in \omega: h_{2 x}(k) \neq 0\right\}$. Now for some $b \in \omega, y=b \cdot 3^{m}+\sum_{k=0}^{m-1} g(k) \cdot 3^{k}$. Thus $l(y+x)=l(y+2 x)=t$. But, as a simple consideration of cases on $g(t)$ and $h_{x}(t)$ shows, $h_{y+x}(t) \neq h_{y+2 x}(t)$, a contradiction.

We also observe that Theorem 2.11 provides a negative answer to a question of Ellis (in unpublished lecture notes). It is a fact [11, Proposition 2.6] that given a compact metric space $X$ and continuous $T: X \rightarrow X$, there exist $x$ and sequence $\left\langle n_{k}\right\rangle_{k=1}^{\infty}$ so that $x=\lim _{k \rightarrow \infty} T^{n_{k}}(x)=\lim _{k \rightarrow \infty} T^{2 n_{k}}(x)$. The question is whether a similar result must hold in any compact Hausdorff space. Since any infinite closed subset of $\beta N$ contains a copy of $\beta N[14,6 \mathscr{O} 6]$ no sequence in $\beta N$ converges unless it is constant. Accordingly, we replace the notion of $\lim _{k \rightarrow \infty} T^{n_{k}}(x)$ with the notion of $p-\lim _{n \in N} T^{n}(x)$ (Definition 2.2). 2.12 Corollary. Define $T: \beta N \rightarrow \beta N$ by $T(p)=p+1$. Let $p, q \in \beta N$. Then $p-\lim _{n \in N} T^{n}(q) \neq p-\lim _{n \in N} T^{2 n}(q)$.

Proof. We show here that $p-\lim _{n \in N} T^{2 n}(q)=q+2 p$ (which is not $q+p$ by 
Theorem 2.11). Indeed, let $A \in q+2 p$. Let $B=\{x \in N: A-x \in q\}$ so that $B \in 2 p$ and hence $B / 2 \in p$. Then $B / 2 \subseteq\left\{n: T^{2 n}(q) \in \bar{A}\right\}$ so $\left\{n: T^{2 n}(q) \in\right.$ $\bar{A}\} \in p$ as required.

Similarly $q+p=p-\lim _{n \in N} T^{n}(q)$.

\section{A SHORT PROOF OF VAN DER WAERDEN'S THEOREM- AN INTRODUCTION TO CENTRAL SETS}

The rest of the results of this paper are based on a simple construction of Furstenberg and Katznelson. Even though a strong version of van der Waerden's Theorem is one of the consequences of the general construction, we present a proof here to introduce the technique without some of the complications involved in the more general results. (Also, we believe this is the simplest proof of van der Waerden's Theorem to date.)

The motivation for the term "central" comes from the definition and results of [11, Chapter 8]. For a discussion of the relationship between these notions see $\S 6$.

3.1 Definition. (a) Let $T$ be a compact left-topological semigroup. A point $p \in T$ is a minimal idempotent if and only if $p$ is an idempotent and there is a minimal right ideal $R$ with $p \in R$.

(b) Let $(S,+)$ be a discrete semigroup. A set $A \subseteq S$ is central in $S$ if and only if there is a minimal idempotent $p$ of $\beta S$ with $A \in p$.

Although we will not use this fact, it is worth noting that the union of all minimal right ideals of a compact left topological semigroup is a two-sided ideal which is the smallest two sided ideal. (See [26] or [5].) This ideal is customarily called the minimal ideal. Thus $p$ is a minimal idempotent if and only if it is an idempotent in the minimal ideal.

It is well known that the relation defined by $q \leq p$ if and only if $q=q+p=$ $p+q$ is a partial order on the idempotents of $T$. (See [5].) It is also well known that an idempotent is in the minimal ideal if and only if it is minimal with respect to this order. Since this latter fact is important to us, and has an easy proof, we shall prove it.

3.2 Lemma. Let $(T,+)$ be a compact left-topological semigroup.

(a) Let $p$ be any idempotent of $T$. There is a minimal idempotent $q$ of $T$ with $q \leq p$.

(b) Let $p$ and $q$ be idempotents of $T$ with $p$ minimal and $q \leq p$. Then $q=p$.

Proof. (a) Since $p+T$ is a right ideal of $T$ it contains a minimal right ideal $R$ which has an idempotent $t$. (Then $R=t+T$.) Let $q=t+p$ and note that $q \in R$. Since $R \subseteq p+T$, pick $r \in T$ such that $t=p+r$. Then $q=p+r+p$. Now $q+q=p+r+p+p+r+p=p+r+p+r+p=t+t+p=t+p=q$ so $q$ is an idempotent. Also $q+p=t+p+p=t+p=q$ and $p+q=p+p+r+p=$ $p+r+p=q$ so $q \leq p$. 
(b) (Of course, had we defined "minimal" in terms of $\leq$ this statement would be a complete triviality.) Pick a minimal right ideal $R$ such that $p \in R$. Since $q=p+q, q \in R$ so $q+T \subseteq R$ so $q+T=R$. Pick $r \in T$ such that $p=q+r$. Then $q=q+p=q+q+r=q+r=p$.

The proof of the following lemma is completely routine and we omit it.

3.3 Lemma. Let $T_{1}$ and $T_{2}$ be compact left topological semigroups. Then $T_{1} \times T_{2}$ with the product topology and coordinatewise operations is a compact left topological semigroup. Further if $x \in T_{1}, y \in T_{2}, \rho_{x}: T_{1} \rightarrow T_{1}$ and $\rho_{y}: T_{2} \rightarrow T_{2}$ are continuous, then $\rho_{(x, y)}: T_{1} \times T_{2} \rightarrow T_{1} \times T_{2}$ is continuous.

We are now ready to outline the general procedure which we will follow in this and the next section. We start with a fixed $l \in N$ and a discrete semigroup $S$ and let $T=(\beta S)^{l}$ (which is by Lemma 3.3 a compact left topological semigroup). One then defines subsets $E^{*}$ and $I^{*}$ of $(S)^{l}$ and lets $E=\operatorname{cl}_{T} E^{*}$ and $I=\mathrm{cl}_{T} I^{*}$. One then shows that $E$ is a (necessarily compact) subsemigroup of $T, I$ is an ideal of $E$, and whenever $p$ is a minimal idempotent of $\beta S$, then $(p, p, \ldots, p) \in E$. Using Lemma 3.2 one shows that necessarily $(p, p, \ldots, p) \in I$. These essential steps (but in the context of enveloping semigroups) are all contained in the paper of Furstenberg and Katznelson [12].

We now display the definitions of $E^{*}$ and $I^{*}$ which are relevant for van der Waerden's Theorem (with the understanding that the definitions will become "inoperative" at the end of the section).

3.4 Definition. Let $l \in N$.

(a) $T=(\beta N)^{l}$ with addition as the operation.

(b) $E^{*}=\{(a, a+d, a+2 d, \ldots, a+(l-1) d): a \in N$ and $d \in \omega\}$.

(c) $I^{*}=\{(a, a+d, a+2 d, \ldots, a+(l-1) d): a, d \in N\}$.

(d) $E=\mathrm{cl}_{T} E^{*}$ and $I=\mathrm{cl}_{T} I^{*}$.

3.5 Lemma. $E$ is a compact left topological semigroup and $I$ is a (two-sided) ideal of $E$.

Proof. The topological conclusions are inherited from $T$. We let $\vec{p}=\left(p_{1}, p_{2}\right.$, $\left.\ldots, p_{l}\right)$ and $\vec{q}=\left(q_{1}, q_{2}, \ldots, q_{l}\right)$ be in $E$ and show that $\vec{p}+\vec{q} \in E$. We show further that if either $\vec{p}$ or $\vec{q}$ is in $I$, then $\vec{p}+\vec{q} \in I$.

To this end let $V_{1} \times V_{2} \times \cdots \times V_{l}$ be a basic neighborhood of $\vec{p}+\vec{q}$. Pick, by left continuity a neighborhood $U_{1} \times U_{2} \times \cdots \times U_{l}$ of $\vec{q}$ such that $\vec{p}+\left(U_{1} \times\right.$ $\left.U_{2} \times \cdots \times U_{l}\right) \subseteq V_{1} \times V_{2} \times \cdots \times V_{l}$. Pick $a \in N$ and $d \in \omega$ (with $d>0$ if $\vec{q} \in I)$ such that $(a, a+d, \ldots, a+(l-1) d) \in U_{1} \times U_{2} \times \cdots \times U_{l}$ and let $\vec{x}=(a, a+d, \ldots, a+(l-1) d)$. Then $\vec{p}+\vec{x} \in V_{1} \times V_{2} \times \cdots \times V_{l}$ and, by Lemma 3.2, $\rho_{\vec{x}}$ is continuous so pick a neighborhood $W_{1} \times W_{2} \times \cdots \times W_{l}$ of $\vec{p}$ with $W_{1} \times W_{2} \times \cdots \times W_{l}+\vec{x} \subseteq V_{1} \times V_{2} \times \cdots \times V_{l}$. Pick $b \in N$ and $e \in \omega$ (with $e>0$ if $\vec{p} \in I)$ such that $(b, b+e, \ldots, b+(l-1) e) \in W_{1} \times W_{2} \times \cdots \times W_{l}$. 
Let $\vec{y}=(b, b+e, \ldots, b+(l-1) e)$. Then $\vec{y}+\vec{x} \in V_{1} \times V_{2} \times \cdots \times V_{l}$, $\vec{y}+\vec{x}=(a+b,(a+b)+(d+e), \ldots,(a+b)+(l-1)(d+e))$, and if either $p \in I$ or $q \in I$, then $d+e>0$.

3.6 Lemma. If $p \in \beta N$ and $\vec{p}=(p, p, \ldots, p)$, then $\vec{p} \in E$.

Proof. Let $U_{1} \times U_{2} \times \cdots \times U_{l}$ be a neighborhood of $\vec{p}$. Then $U_{1} \cap U_{2} \cap \cdots \cap U_{l}$ is a neighborhood of $p$ so pick $a \in N \cap U_{1} \cap U_{2} \cap \cdots \cap U_{l}$. Then $(a, a, \ldots, a) \in$ $\left(U_{1} \times U_{2} \times \cdots \times U_{l}\right) \cap E^{*}$.

3.7 Theorem. Let $p$ be a minimal idempotent in $\beta N$ and let $\vec{p}=(p, p, \ldots$, p). Then $\vec{p} \in I$.

Proof. We have by Lemma 3.6 that $\vec{p} \in E$ so $\vec{p}$ is an idempotent in $E$. Pick by Lemma 3.2 (a) a minimal idempotent $\vec{q}$ of $E$ with $\vec{q} \leq \vec{p}$. Write $\vec{q}=\left(q_{1}, q_{2}, \ldots, q_{l}\right)$. Then $\vec{q}=\vec{q}+\vec{p}=\vec{p}+\vec{q}$ so for each $i \in\{1,2, \ldots, l\}$, $q_{i}=q_{i}+p=p+q_{i}$ so that by Lemma 3.2(b), $q_{i}=p$. Thus $\vec{q}=\vec{p}$ so that $\vec{p}$ is a minimal idempotent of $E$. Pick a minimal right ideal $R$ of $E$ with $\vec{p} \in R$. Since $I$ is a left ideal of $E, I \cap R \neq \varnothing$. (Indeed, pick $\vec{x} \in I$ and $\vec{y} \in R$. Then $\vec{y}+\vec{x} \in I \cap R$.) Since $I$ is a right ideal of $E$, and $\varnothing \neq I \cap R \subseteq R$, $I \cap R=R$. But then $\vec{p} \in I$ as required.

3.8 Corollary. Let $A$ be a central set in $N$. Then $A$ contains an $l$ term arithmetic progression. In particular some element of any finite partition of $N$ contains an l term arithmetic progression.

Proof. Pick a minimal idempotent $p$ in $\beta N$ with $A \in p$. Let $\vec{p}=(p, p, \ldots$, $p)$. Then $\vec{p} \in I$ and $\bar{A} \times \bar{A} \times \cdots \times \bar{A}$ is a neighborhood of $\vec{p}$ so pick $a, d \in N$ with $(a, a+d, a+2 d, \ldots, a+(l-1) d) \in \bar{A} \times \bar{A} \times \cdots \times \bar{A}$.

\section{Central sets in Semigroups}

Using the methods from $\S 3$ we produce here a version of [11, Proposition 9.21] which is applicable to a wide class of semigroups. For convenience, we give this class a name.

4.1 Definition. A pre-natural semigroup is a triple $(S,+, \varphi)$ where $(S,+)$ is a commutative semigroup and $\varphi$ is a homomorphism from $(S,+)$ to $(N,+)$.

Examples of pre-natural semigroups include $(N,+, l),(N \backslash\{1\}, \cdot, \varphi)$ (where $\varphi(x)$ is the length of the prime factorization of $x$ ), and the nonconstant polynomials over any integral domain under multiplication (where $\varphi(P)=$ degree of $P)$. Also, if $\left(S_{1},+, \varphi_{1}\right)$ and $\left(S_{2},+, \varphi_{2}\right)$ are pre-natural so is $\left(S_{1} \times S_{2},+, \varphi\right)$ (where $\left.\varphi(x, y)=\varphi_{1}(x)+\varphi_{2}(y)\right)$. Finally observe that if $\left(S_{1},+, \varphi_{1}\right)$ is a pre-natural semigroup, $\left(S_{2},+\right)$ is a commutative semigroup, and $\varphi_{2}$ is a homomorphism from $S_{2}$ to $S_{1}$, then $\left(S_{2},+, \varphi_{1} \circ \varphi_{2}\right)$ is a prenatural semigroup. Consequently, the Gaussian integers with norm greater than 
1 from a pre-natural semigroup under multiplication, since \|\| is a homomorphism to $(N \backslash\{1\}, \cdot)$.

Note that, since $0 \notin N$, a pre-natural semigroup cannot have an identity, not even an idempotent.

We have already defined $F S(B)$ for $B \subseteq N$ and the same definition applies to any commutative semigroup. Given a sequence $\left\langle x_{n}\right\rangle_{n=1}^{\infty}$ in a commutative semigroup $(S,+)$ we define analogously $F S\left(\left\langle x_{n}\right\rangle_{n=1}^{\infty}\right)=\left\{\sum_{n \in F} x_{n}: F\right.$ is a finite nonempty subset of $N\}$. (So, if $\left\langle x_{n}\right\rangle_{n=1}^{\infty}$ is one-to-one, $F S\left(\left\langle x_{n}\right\rangle_{n=1}^{\infty}\right)=$ $F S\left(\left\{x_{n}: n \in N\right\}\right)$.)

4.2 Definition. A sequence $\left\langle x_{n}\right\rangle_{n=1}^{\infty}$ in a pre-natural semigroup $(S,+, \varphi)$ is thin provided whenever $m, r \in N$ with $m<r$ and $t \in \omega$, if $2^{t} \leq \varphi\left(x_{m}\right)$, then $2^{t+1} \mid \varphi\left(x_{r}\right)$.

4.3 Lemma. Let $\left\langle x_{n}\right\rangle_{n=1}^{\infty}$ be a sequence in a pre-natural semigroup $(S,+, \varphi)$.

(a) There exists a sequence $\left\langle F_{m}\right\rangle_{m=1}^{\infty}$ of pairwise disjoint finite nonempty subsets of $N$ such that for all $m, \max F_{m}<\min F_{m+1}$ and $\left\langle\sum_{n \in F_{m}} x_{n}\right\rangle_{m=1}^{\infty}$ is thin.

(b) If $\left\langle x_{n}\right\rangle_{n=1}^{\infty}$ is thin and $F$ is a finite nonempty subset of $N$ and $2^{t} \mid \varphi\left(\sum_{m \in F} x_{m}\right)$, then for each $m \in F, 2^{t} \mid \varphi\left(x_{m}\right)$.

Proof. (a) We construct the sequence $\left\langle F_{m}\right\rangle_{m=1}^{\infty}$ inductively. Let $F_{1}=\{1\}$. Given $\left\langle F_{i}\right\rangle_{i=1}^{m}$, let $t \in \omega$ be the largest such that $2^{t} \leq \varphi\left(\sum_{n \in F_{m}} x_{n}\right)$. Choose by the pigeon hole principle $a \in\left\{0,1,2, \ldots, 2^{t+1}\right\}$ and a subset $F_{m+1} \subseteq\{n \in$ $\left.N: n>\max F_{m}\right\}$ such that $\left|F_{m+1}\right|=2^{t+1}$ and for each $n \in F_{m+1}, \varphi\left(x_{n}\right) \equiv a$ $\left(\bmod 2^{t+1}\right)$. Then $\varphi\left(\sum_{n \in F_{m+1}} x_{n}\right) \equiv 0\left(\bmod 2^{t+1}\right)$ as required.

(b) For this one simply observes that if $n<m$ and $\varphi\left(x_{n}\right)$ and $\varphi\left(x_{m}\right)$ are written in binary, their supports are disjoint and hence no carrying occurs when they are added.

We need to modify the style of the construction of $\S 3$ somewhat by restricting ourselves to a semigroup of $\beta S$.

4.4 Definition. Given a pre-natural semigroup $(S,+, \varphi)$ and $n \in N, X_{n}=$ $\left\{x \in S: 2^{n} \mid \varphi(x)\right\}$.

Observe that, as a consequence of the existence of thin sequences (Lemma 4.3(a)) each $X_{n} \neq \varnothing$.

The following lemma is well known, although we have not found an explicit statement in the literature.

4.5 Lemma. Let $(S,+)$ be a commutative discrete semigroup and let $p \in$ $\beta S$ with $p+p=p$. Given any $A \in p$ there is a sequence $\left\langle x_{n}\right\rangle_{n=1}^{\infty}$ with $F S\left(\left\langle x_{n}\right\rangle_{n=1}^{\infty}\right) \subseteq A$.

Proof. Galvin's original argument presented in [18, Theorem 3.3] applies. 
4.6 Lemma. Let $(S,+, \varphi)$ be a pre-natural semigroup and let $n$ be an arbitrary member of $N$. Then $\bar{X}_{n}$ is a (compact) subsemigroup of $\beta S$. Further each idempotent of $\beta S$ is in $\bar{X}_{n}$.

Proof. Let $p, q \in \bar{X}_{n}$. To see that $X_{n} \in p+q$, we show $X_{n} \subseteq\left\{x \in S: X_{n}-x \in\right.$ $p$ \}. To this end, let $x \in X_{n}$. We claim $X_{n} \subseteq X_{n}-x$. Let $y \in X_{n}$. Then $\varphi(y+x)=\varphi(y)+\varphi(x), 2^{n} \mid \varphi(y)$, and $2^{n} \mid \varphi(x)$ so $2^{n} \mid \varphi(y+x)$ as required.

Now let $p \in \beta S$ with $p+p=p$.

Suppose $X_{n} \notin p$. Then $S \backslash X_{n} \in p$ so pick a sequence $\left\langle x_{m}\right\rangle_{m=1}^{\infty}$ with $F S\left(\left\langle x_{m}\right\rangle_{m=1}^{\infty}\right) \subseteq S \backslash X_{n}$. As in the proof of Lemma 4.3, pick $F \subseteq N$ such that $\left.2^{n} \mid \varphi \sum_{m \in F} x_{m}\right)$. Then $\sum_{m \in F} x_{m} \in F S\left(\left\langle x_{m}\right\rangle_{m=1}^{\infty}\right) \cap X_{n}$, a contradiction.

As in $\S 3$ we make a local definition of $E^{*}, I^{*}, E$, and $I$, to remain in effect through Theorem 4.11. (Also, as in $\S 3$, the notation does not express the dependence on $l$, the choice of $(S,+, \varphi)$, and the choice of $\left\langle y_{i, n}\right\rangle_{n=1}^{\infty}$.)

4.7 Definition. Let $l \in N$, let $(S,+, \varphi)$ be a pre-natural semigroup, and for each $i \in\{1,2, \ldots, l\}$, let $\left\langle y_{i, n}\right\rangle_{n=1}^{\infty}$ be a sequence in $S$ such that $\left\langle y_{1, n}\right\rangle_{n=1}^{\infty}$ is thin.

(a) $T=(\beta S)^{l+1}$.

(b) $I^{*}=\left\{\left(a, a+\sum_{n \in F} y_{1, n}, a+\sum_{n \in F} y_{2, n}, \ldots, a+\sum_{n \in F} y_{l, n}\right): F\right.$ is a finite nonempty subset of $N$ and $a \in S\}$.

(c) $E^{*}=I^{*} \cup\{(a, a, \ldots, a): a \in S\}$.

(d) $I=\bigcap_{n=1}^{\infty} \operatorname{cl}_{T}\left(X_{n}^{l+1} \cap I^{*}\right)$.

(e) $E=\bigcap_{n=1}^{\infty} \mathrm{cl}_{T}\left(X_{n}^{l+1} \cap E^{*}\right)$.

Unlike the situation in $\S 3$, it is no longer obvious that $I \neq \varnothing$.

\subsection{Lemma. $I \neq \varnothing$.}

Proof. Since $T$ is compact and $I=\bigcap_{n=1}^{\infty} \mathrm{cl}_{T}\left(X_{n}^{l+1} \cap I^{*}\right)$, it suffices to let $n \in N$ and show $X_{n}^{l+1} \cap I^{*} \neq \varnothing$. (Note that each $X_{n+1} \subseteq X_{n}$.) Pick $a \in X_{n}$. It is enough to produce finite nonempty $F \subseteq N$ such that for each $i \in\{1,2, \ldots, l\}, 2^{n} \mid \varphi\left(\sum_{m \in F} y_{i, m}\right)$. For then $2^{n} \mid \varphi\left(a+\sum_{m \in F} y_{i, m}\right)$ so that $\left(a, a+\sum_{m \in F} y_{1, m}, a \in \sum_{m \in F} y_{2, m}, \ldots, a+\sum_{m \in F} y_{l, m}\right) \in\left(X_{n}\right)^{l+1} \cap I^{*}$.

We show this can be done by induction on $l$. If $l=1$, since $\left\langle y_{1, m}\right\rangle_{m=1}^{\infty}$ is thin, $F=\{n+1\}$ will do the job. Assume $l>1$ and, applying the induction hypotheses $2^{2 n}$ times, produce pairwise disjoint $\left\langle F_{t}\right\rangle_{t=1}^{2^{2 n}}$ such that for each $t \in\left\{1,2, \ldots, 2^{2 n}\right\}$ and each $i \in\{1,2, \ldots, l-1\}, 2^{n} \mid \varphi\left(\sum_{m \in F_{l}} y_{i, m}\right)$. Choose by the pigeonhole principle, $G \subseteq\left\{1,2, \ldots, 2^{2 n}\right\}$ with $|G|=2^{n}$ and for $t$, $s \in G,\left(\sum_{m \in F_{t}} y_{l, m}\right) \equiv \varphi\left(\sum_{m \in F_{s}} y_{l, m}\right)\left(\bmod 2^{n}\right)$. Let $F=\bigcup_{t \in G} F_{t}$.

4.9 Lemma. $E$ is a compact left topological semigroup and $I$ is an ideal of E.

Proof. Let $\vec{p}=\left(p_{0}, p_{1}, \ldots, p_{l}\right)$ and $\vec{q}=\left(q_{0}, q_{1}, \ldots, q_{l}\right)$ be in $E$. We show that $\vec{p}+\vec{q} \in E$ and, if $\vec{p}$ or $\vec{q}$ is in $I$, then $\vec{p}+\vec{q} \in I$. For notational 
convenience we agree that $a+\sum_{n \in \varnothing} x_{n}=a$ for any $a \in S$ and any sequence $\left\langle x_{n}\right\rangle_{n=1}^{\infty}$ in $S$.

Let $s \in N$. We show $\vec{p}+\vec{q} \in \mathrm{cl}_{T}\left(X_{s}^{l+1} \cap E^{*}\right)$ and, if $\vec{p}$ or $\vec{q}$ is in $I$ then $\vec{p}+\vec{q} \in \operatorname{cl}_{T}\left(X_{s}^{l+1} \cap I^{*}\right)$. Let $V_{0} \times V_{1} \times \cdots \times V_{l}$ be a basic neighborhood of $\vec{p}+\vec{q}$. Pick by left continuity a neighborhood $U_{0} \times U_{1} \times \cdots \times U_{l}$ of $\vec{q}$ such that $\vec{p}+\left(U_{0} \times U_{1} \times \cdots \times U_{l}\right) \subseteq V_{0} \times V_{1} \times \cdots \times V_{l}$. Pick $a \in S$ and a finite subset $F$ of $N$ (with $F \neq \varnothing$ if $\vec{q} \in I$ ) such that $\left(a, a+\sum_{n \in F} y_{1, n}, a+\sum_{n \in F} y_{2, n}\right.$, $\left.\cdots, a+\sum_{n \in F} y_{l, n}\right) \in\left(U_{0} \times U_{1} \times \cdots \times U_{l}\right) \cap X_{s}^{l+1}$. Let $\vec{x}=\left(a, a+\sum_{n \in F} y_{1, n}\right.$, $\left.a+\sum_{n \in F} y_{2, n}, \ldots, a+\sum_{n \in F} y_{l, n}\right)$. Let $m=\max F$ (or $m=1$ if $F=\varnothing$ ) and let $t$ be the largest integer with $2^{t} \leq \varphi\left(y_{1, m}\right)$ and let $r=t+1$. By continuity of $\rho_{\vec{x}}$, pick a neighborhood $W_{0} \times W_{1} \times \cdots \times W_{l}$ of $\vec{p}$ with $\left(W_{0} \times W_{1} \times \cdots \times W_{l}\right)+\vec{x} \subseteq$ $V_{0} \times V_{1} \times \cdots \times V_{l}$. Since $\vec{p} \in \operatorname{cl}_{T}\left(X_{r}^{l+1} \cap E^{*}\right)$, pick $b \in S$ and a finite subset $G$ of $N$ (with $G \neq \varnothing$ if $p \in I$ ) such that $\left(b, b+\sum_{n \in G} y_{1, n}, b+\sum_{n \in G} y_{2, n}\right.$, $\left.\ldots, b+\sum_{n \in G} y_{l, n}\right) \in W_{0} \times W_{1} \times \cdots \times W_{l}$ and such that $b \in X_{r}$ and for each $i \in\{1,2, \ldots, l\}, b+\sum_{n \in G} y_{i, n} \in X_{r}$. Let $\vec{y}=\left(b, b+\sum_{n \in G} y_{1, n}\right.$, $\left.b+\sum_{n \in G} y_{2, n}, \ldots, b+\sum_{n \in G} y_{l, n}\right)$. Then $\vec{y}+\vec{x} \in\left(V_{0} \times V_{1} \times \cdots \times V_{l}\right) \cap X_{s}^{l+1}$. It suffices to show $F \cap G=\varnothing$. For then, letting $H=F \cup G, \vec{y}+\vec{x}=(b+a$, $\left.b+a+\sum_{n \in H} y_{1, n}, b+a+\sum_{n \in H} y_{2, n}, \ldots, b+a+\sum_{n \in H} y_{l, n}\right)$ and if either $F \neq \varnothing$ or $G \neq \varnothing$, then $H \neq \varnothing$.

To this end, we may assume $F \neq \varnothing$ and $G \neq \varnothing$. Now $2^{r} \mid \varphi(b)$ and $2^{r} \mid \varphi\left(b+\sum_{n \in G} y_{1, n}\right)$ so $2^{r} \mid \varphi\left(\sum_{n \in G} y_{1, n}\right)$. Let $k=\min G$. Then, since $\left\langle y_{1, n}\right\rangle_{n=1}^{\infty}$ is a thin sequence $2^{r} \mid \varphi\left(y_{1, k}\right)$ by Lemma $4.3(\mathrm{~b})$. Since $2^{r}>\varphi\left(y_{1, m}\right)$ we have $\max F=m<k=\min G$.

4.10 Lemma. Let $p \in \beta S$ with $p+p=p$ and let $\vec{p}=(p, p, \ldots, p)$. Then $p \in E$. If $p$ is minimal in $\beta S$, then $\vec{p} \in I$.

Proof. Let $n \in N$ and let $U_{0} \times U_{1} \times \cdots \times U_{l}$ be a neighborhood of $\vec{p}$. Then $U_{0} \cap U_{1} \cap \cdots \cap U_{l}$ is a neighborhood of $p$ while, by Lemma $4.6 p \in \bar{X}_{n}$. Let $a \in U_{0} \cap U_{1} \cap \cdots \cap U_{l} \cap X_{n}$. Then $(a, a, \ldots, a) \in\left(U_{0} \times U_{1} \times \cdots \times U_{l}\right) \cap\left(X_{n}^{l+1} \cap E^{*}\right)$.

The proof the second assertion is identical to the proof of Lemma 3.7.

The following theorem, the major result of this section, corresponds to Proposition 8.21 of [11] (except in one detail-see Theorem 4.12).

4.11 Theorem. Let $l \in N$, let $(S,+, \varphi)$ be a pre-natural semigroup, and for each $i \in\{1,2, \ldots, l\}$, let $\left\langle y_{i, n}\right\rangle_{n=1}^{\infty}$ be a sequence in $S$. Let $A$ be a central set in $S$. There exist a sequence $\left\langle a_{n}\right\rangle_{n=1}^{\infty}$ in $S$ and a sequence $\left\langle H_{n}\right\rangle_{n=1}^{\infty}$ of pairwise disjoint finite nonempty subsets of $N$ such that, whenever $\stackrel{n}{F}$ is a finite nonempty subset of $N$ one has $\left(\sum_{n \in F} a_{n}, \sum_{n \in F}\left(a_{n}+\sum_{m \in H_{n}} y_{1, m}\right)\right.$, $\left.\sum_{n \in F}\left(a_{n}+\sum_{m \in H_{n}} y_{2, m}\right), \ldots, \sum_{n \in F}\left(a_{n}+\sum_{m \in H_{n}} y_{l, m}\right)\right) \in A^{l+1}$. 
Proof. It suffices to prove the theorem under the additional assumption that $\left\langle y_{1, n}\right\rangle_{n=1}^{\infty}$ is a thin sequence. (For then, choose a sequence by Lemma 4.3(a) $\left\langle F_{n}\right\rangle_{n=1}^{\infty}$ such that $\left\langle\sum_{m \in F_{n}} y_{1, m}\right\rangle_{n=1}^{\infty}$ is thin and let for each $i$ and $n, y_{i, n}^{\prime}=$ $\sum_{m \in F_{n}} y_{i, m}$. Given $\left\langle a_{n}\right\rangle_{n=1}^{\infty}$ and $\left\langle H_{n}^{\prime}\right\rangle_{n=1}^{\infty}$ working for $\left\langle y_{i, n}^{\prime}\right\rangle_{n=1}^{\infty}$ we let $H_{n}=$ $\bigcup_{t \in H_{n}^{\prime}} F_{t}$. Then $\left.a_{n}+\sum_{m \in H_{n}} y_{i, n}=a_{n}+\sum_{t \in H_{n}^{\prime}} \sum_{m \in F_{t}} y_{i, m}=a_{n}+\sum_{t \in H_{n}^{\prime}} y_{i, t}^{\prime}.\right)$ Accordingly we do assume that $\left\langle y_{1, n}\right\rangle_{n=1}^{\infty}$ is a thin sequence.

Let $T, I^{*}, E^{*}, I$, and $E$ be as in Definition 4.7. Pick a minimal idempotent $p \in \beta S$ with $A \in p$ and let $\vec{p}=(p, p, \ldots, p)$. By Lemma $4.10, \vec{p} \in I$.

Let $A_{1}=A$. Since $A_{1} \in p$, we have $\left(\bar{A}_{1}\right)^{l+1}$ is a neighborhood of $\vec{p}=$ $\vec{p}+\vec{p}$. Pick by left continuity a neighborhood $U_{1}$ of $\vec{p}$ with $\vec{p}+U_{1} \subseteq\left(\bar{A}_{1}\right)^{l+1}$. Pick $B_{1} \in p$ with $\left(\bar{B}_{1}\right)^{l+1} \subseteq U_{1}$ and $B_{1} \subseteq A_{1}$. Then $\left(\bar{B}_{1}\right)^{l+1} \cap I^{*} \neq \varnothing$ so pick $\vec{x}_{1} \in\left(\bar{B}_{1}\right)^{l+1} \cap I^{*}$ and pick $a_{1} \in S$ and finite nonempty $H_{1} \subseteq N$ such that $\vec{x}_{1}=\left(a_{1}, a_{1}+\sum_{m \in H_{1}} y_{1, m}, \ldots, a_{n}+\sum_{m \in H_{1}} y_{l, m}\right)$. Let $t_{1}$ be the largest integer such that $2^{t_{1}} \leq \varphi\left(a_{1}+\sum_{m \in H_{1}} y_{1, m}\right)$. Since $\vec{p}+\vec{x}_{1} \in\left(\bar{A}_{1}\right)^{l+1}$ and $\rho_{\vec{x}_{1}}$ is continuous, pick a neighborhood $V_{1}$ of $\vec{p}$ with $V_{1}+\vec{x}_{1} \subseteq\left(\bar{A}_{1}\right)^{l+1}$. Pick $A_{2} \in p$ with $A_{2} \subseteq X_{t_{1}+1} \cap B_{1}$ and $\left(\bar{A}_{2}\right)^{l+1} \subseteq V_{1}$.

At step $n$ of the induction, having chosen $A_{n}, B_{n-1}, \vec{x}_{n-1}, a_{n-1}, U_{n-1}$, $V_{n-1}, H_{n-1}$ and $t_{n-1}$, we proceed in an identical fashion. That is, we have $\left(\bar{A}_{n}\right)^{l+1}$ is a neighborhood of $\vec{p}=\vec{p}+\vec{p}$ so we pick a neighborhood $U_{n}$ of $\vec{p}$ with $\vec{p}+U_{n} \subseteq\left(\bar{A}_{n}\right)^{l+1}$. We pick $B_{n} \in p$ with $\left(\bar{B}_{n}\right)^{l+1} \subseteq U_{n}$ and $B_{n} \subseteq A_{n}$. We pick $\vec{x}_{n} \in\left(\bar{B}_{n}\right)^{l+1} \cap I^{*}$ and choose $a_{n} \in S$ and finite nonempty $H_{n} \subseteq N$ with $\vec{x}_{n}=\left(a_{n}, a_{n}+\sum_{m \in H_{n}} y_{1, n}, \ldots, a_{n}+\sum_{m \in H_{n}} y_{l, m}\right)$. We let $t_{n}$ be the largest integer with $2^{t_{n}} \leq \varphi\left(a_{n}+\sum_{m \in H_{n}} y_{1, m}\right)$. By the continuity of $\rho_{\vec{x}_{n}}$ we pick a neighborhood $V_{n}$ of $\vec{p}$ with $V_{n}+\vec{x}_{n} \subseteq\left(\bar{A}_{n}\right)^{l+1}$ and we pick $A_{n+1} \in p$ with $A_{n+1} \subseteq X_{t_{n}+1} \cap B_{n}$ and $\left(\overline{A_{n+1}}\right)^{l+1} \subseteq V_{n}$.

Observe that $\max H_{n-1}<\min H_{n}$. Indeed, let $k=\max H_{n-1}$ and let $t=$ $t_{n-1}$. Now

$$
\vec{x}_{n} \in\left(B_{n}\right)^{l+1} \subseteq\left(X_{t+1}\right)^{l+1} .
$$

Thus $2^{t+1} \mid \varphi\left(a_{n}\right)$ and $2^{t+1} \mid \varphi\left(a_{n}+\sum_{m \in H_{n}} y_{1, m}\right)$ so $2^{t+1} \mid \varphi\left(\sum_{m \in H_{n}} y_{1, m}\right)$. Thus, by Lemma 4.3(b) for each $m \in H_{n} 2^{t+1} \mid \varphi\left(y_{1, m}\right)$. Since

$$
2^{t+1}>\varphi\left(a_{n-1}+\sum_{m \in H_{n-1}} y_{1, m}\right)>\varphi\left(y_{1, k}\right)
$$

we have $k<\min H_{n}$ as required.

We finish the proof by showing that $\left\langle a_{n}\right\rangle_{n=1}^{\infty}$ and $\left\langle H_{n}\right\rangle_{n=1}^{\infty}$ satisfy the conclusion of the theorem. For this we show by induction on $|F|$, that if $r=\min F$, then $\sum_{n \in F} \vec{x}_{n} \in\left(A_{r}\right)^{l+1}$. If $|F|=1$, then $\sum_{n \in F} \vec{x}_{n}=\vec{x}_{r} \in\left(B_{r}\right)^{l+1} \subseteq\left(A_{r}\right)^{l+1}$. 
Assume $|F|>1$, let $r=\min F$, let $G=F \backslash\{r\}$, and let $s=\min G$. Then $\sum_{n \in G} \vec{x}_{n} \in\left(A_{s}\right)^{l+1}$ by our inductive hypothesis. Since $A_{s} \subseteq A_{s-1} \subseteq \cdots \subseteq$ $A_{r+1}$, we have $\sum_{n \in G} \vec{x}_{n} \in\left(A_{r+1}\right)^{l+1} \subseteq V_{r}$. Since $V_{r}+\vec{x}_{r} \subseteq\left(\vec{A}_{r}\right)^{l+1}$ we have $\sum_{n \in G} \vec{x}_{n}+\vec{x}_{r} \in\left(A_{r}\right)^{l+1}$ as required.

Proposition 8.21 of [11] is stronger than the case of Theorem 4.11 with $S=N$ since the sequences are allowed to come from $Z$. We indicate now how to obtain this result by our methods (and for our version of "central" sets).

4.12 Theorem. Let $l \in N$ and for each $i \in\{1,2, \ldots, l\}$ let $\left\langle y_{i, n}\right\rangle$ be a sequence in $Z$. Let $A$ be a central set in $N$. There exist a sequence $\left\langle a_{n}\right\rangle_{n=1}^{\infty}$ in $N$ and a sequence $\left\langle H_{n}\right\rangle_{n=1}^{\infty}$ of pairwise disjoint finite nonempty subsets of $N$ such that, whenever $F$ is a finite nonempty subset of $N$ one has $\left(\sum_{n \in F} a_{n}\right.$, $\left.\sum_{n \in F}\left(a_{n}+\sum_{m \in H_{n}} y_{1, m}\right), \ldots, \sum_{n \in F}\left(a_{n}+\sum_{m \in H_{n}} y_{l, m}\right)\right) \in A^{l+1}$.

Proof. We can presume that whenever $2^{n} \leq\left|y_{1, m}\right|$, then $2^{n+1} \mid y_{1, m+1}$ and that all terms of $\left\langle y_{1, m}\right\rangle_{m=1}^{\infty}$ have the same sign. (That is essentially that $\left\langle y_{1, m}\right\rangle_{m=1}^{\infty}$ is “thin.") We now define $T=(\beta N)^{l+1}, I^{*}=\left\{\left(a, a+\sum_{n \in F} y_{1, n}, a+\sum_{n \in F} y_{2, n}\right.\right.$, $\left.\ldots, a+\sum_{n \in F} y_{l, n}\right): a \in N, F$ is a finite nonempty subset of $N$, and for each $\left.i \in\{1,2, \ldots, l\}, a+\sum_{n \in F} y_{i, n} \in N\right\}, E^{*}=I^{*} \cup\{(a, a, \ldots, a): a \in N\}$, $I=\bigcap_{n=1}^{\infty} \mathrm{cl}_{T}\left(\left(N 2^{n}\right)^{l+1} \cap I^{*}\right)$ and $E=\left(\bigcap_{n=1}^{\infty} \mathrm{cl}_{T}\left(\left(N 2^{n}\right)^{l+1} \cap E^{*}\right)\right.$. To see, for example, that $I^{*} \cap N 2^{n} \neq \varnothing$, one gets, as in Lemma 4.8, $F \subseteq N$ such that for each $i \in\{1,2, \ldots, l\}, 2^{n} \mid \sum_{m \in F} y_{i, m}$. One then picks $a$ such that $2^{n} \mid a$ and for each $i, a+\sum_{m \in F} y_{i, m}>0$. No major adjustments are required for the rest of the proof.

It would be nicer if, for example, $(N, \cdot)$ were a pre-natural semigroup (or if the set of all nonzero polynomials over an integral domain were a pre-natural semigroup under multiplication). We conclude this section with two corollaries to Theorem 4.11 which do allow us to work with such semigroups which are almost pre-natural.

4.13 Corollary. Let $(S,+)$ be a commutative semigroup and assume that there exist $J \subseteq S$ and $\varphi: J \rightarrow N$ so that $(J,+, \varphi)$ is a pre-natural semigroup and $J$ is an ideal of $S$. Let $l \in N$ and for each $i \in\{1,2, \ldots, l\}$ let $\left\langle y_{i, n}\right\rangle$ be a sequence in $J$. Let $A$ be a central set in $S$. There exist a sequence $\left\langle a_{n}\right\rangle_{n=1}^{\infty}$ in $J$ and a sequence $\left\langle H_{n}\right\rangle_{n=1}^{\infty}$ of pairwise disjoint finite nonempty subsets of $N$ such that, whenever $F$ is a finite nonempty subset of $N$ one has $\left(\sum_{n \in F} a_{n}, \sum_{n \in F}\left(a_{n}+\sum_{m \in H_{n}} y_{1, m}\right), \sum_{n \in F}\left(a_{n}+\sum_{m \in H_{n}} y_{2, m}\right), \ldots\right.$, $\left.\sum_{n \in F}\left(a_{n}+\sum_{m \in H_{n}} y_{l, m}\right)\right) \in A^{l+1}$.

Proof. By Theorem 4.11, it is enough to show that $A \cap J$ is central in $J$. To this end pick a minimal right ideal $R$ of $\beta S$ and $p \in R$ with $p+p=p$ such that $A \in p$. Define $\tau: \bar{J} \rightarrow \beta J$ by $\tau(q)=\{B \cap J: B \in q\}$. It is routine to verify that $\tau$ is an isomorphism and a homeomorphism. It thus suffices to show 
that $R$ is a minimal right ideal of $\bar{J}$. (For then $\tau[R]$ is a minimal ideal of $\beta J, \tau(\rho)$ is an idempotent in $\tau[R]$, and $A \cap J \in \tau(\rho)$.)

We claim that $\bar{J}$ is an ideal of $\beta S$. To see this, let $q \in \beta S$ and $r \in \bar{J}$. Then $S=\{x \in S: J-x \in r\}$ so $J \in r+q$ while $J \subseteq\{x \in S: J-x \in q\}$ so $J \in q+r$.

Since $\bar{J}$ is an ideal and $R$ is a right ideal, $\bar{J} \cap R \neq \varnothing$ and hence $\bar{J} \cap R$ is a right ideal of $\bar{J}$. Pick a minimal right ideal $R^{*}$ of $\bar{J}$ with $R^{*} \subseteq \bar{J} \cap R$. By [4, II.1.8] $R^{*}$ is a right ideal of $\beta S$ and hence $R^{*}=R$ so $R \subseteq \bar{J}$ and $R$ is a minimal right ideal of $\bar{J}$ as required.

Our last corollary applies to any pre-natural semigroup with an identity adjoined-most conspicuously to $(N, \cdot)$. It allows exactly the same conclusion as Theorem 4.11 with only the additional assumption that our hypothesized sequences are one-to-one.

4.14 Corollary. Let $(S,+)$ be a commutative semigroup and assume that there exist $J \subseteq S$ and $\varphi: J \rightarrow N$ so that $(J,+, \varphi)$ is a pre-natural semigroup, $J$ is an ideal of $S$, and $|S \backslash J|<\omega$. Let $l \in N$ and for each $i \in\{1,2, \ldots, l\}$, let $\left\langle y_{i, n}\right\rangle_{n=1}^{\infty}$ be a one-to-one sequence in $S$. Let $A$ be a central set in $S$. There exist a sequence $\left\langle a_{n}\right\rangle_{n=1}^{\infty}$ in $J$ and a sequence $\left\langle H_{n}\right\rangle_{n=1}^{\infty}$ of pairwise disjoint finite nonempty subsets of $N$ such that, whenever $F$ is a finite nonempty subset of $N$ one has $\left(\sum_{n \in F} a_{n}, \sum_{n \in F}\left(a_{n}+\sum_{m \in H_{n}} y_{1, m}\right), \sum_{n \in F}\left(a_{n}+\sum_{m \in H_{n}} y_{2, m}\right), \ldots\right.$, $\left.\sum_{n \in F}\left(a_{n}+\sum_{m \in H_{n}} y_{l, m}\right)\right) \in A^{l+1}$.

Proof. There is some $k \in N$ such that for each $i \in\{1,2, \ldots, l\},\left\langle y_{i, n}\right\rangle_{n=k}^{\infty}$ is a sequence in $J$ so Corollary 4.13 applies.

\section{Properties of Central Sets}

We derive here some consequences of Theorem 4.11. We first observe that an analogue of van der Waerden's Theorem holds in any pre-natural semigroup $S$. Defining for $x \in S$ and $n \in N, n x$ as usual to be $x+x+\cdots+x$ ( $n$ times) one has that any central set $A \subseteq S$ has, for each $l \in N$, some $a, d \in S$ with $\{a, a+d, \ldots, a+l d\} \subseteq A$. (We remind the reader that trivially any finite partition of $S$ contains at least one central set.) Indeed let $\left\langle x_{n}\right\rangle_{n=1}^{\infty}$ be any sequence in $S$ and for $i \in\{1,2, \ldots, l\}$, let $y_{i, n}=i x_{n}$. Pick $\left\langle a_{n}\right\rangle_{n=1}^{\infty}$ and $\left\langle H_{n}\right\rangle_{n=1}^{\infty}$ as guaranteed by Theorem 4.11. Let any finite nonempty subset $F$ of $N$ be given, let $a=\sum_{n \in F} a_{n}$ and $d=\sum_{n \in F} \sum_{m \in H_{n}} x_{m}$. Then $\{a, a+d, \ldots, a+l d\} \subseteq A$. In the case of $(N \backslash\{1\}, \cdot)$, this yields arbitrarily long geometric progressions with common ratio chosen from any pre-designated $F P\left(\left\langle x_{n}\right\rangle_{n=1}^{\infty}\right)$ lying in any central set.

Given any $m \in N,\left(\omega^{m} \backslash\{\overrightarrow{0}\},+, \varphi\right)$, where $\varphi\left(a_{1}, a_{2}, \ldots, a_{m}\right)=\sum_{i=1}^{m} a_{i}$, is a pre-natural semigroup. Using this fact we obtain an $m$-dimensional version of van der Waerden's Theorem due to Grunwald (in [25]). For example, with $m=2$ let $l=8$ and for each $n \in N$ let $x_{1, n}=(n, 0), x_{2, n}=(2 n, 0)$, 
$x_{3, n}=(0, n) x_{4, n}=(0,2 n), x_{5, n}=(n, n), x_{6, n}=(n, 2 n), x_{7, n}=(2 n, n)$, and $x_{8, n}=(2 n, 2 n)$. Then applying Theorem 4.11 we get that each central set in $\omega^{2} \backslash\{(0,0)\}$ contains a lattice as drawn:

$$
\begin{array}{ccc}
(a, b+2 d) & (a+d, b+2 d) & (a+2 d, b+2 d) \\
\cdot & \cdot & \cdot \\
(a, b+d) & (a+d, b+d) & (a+2 d, b+d) \\
\cdot & \cdot & \cdot \\
(a, b) & (a+d, b) & (a+2 d, b)
\end{array}
$$

Likewise using $l=15$, one gets $4 \times 4$ lattices and so on. (Of course Theorem 4.11 applied here says more. It yields a system of lattices and all of their sums.)

We now turn our attention to deriving a version of Deuber's $(m, p, c)$-sets Theorem $([6]$, or see $[15, \S 3.3])$. The $(m, p, c)$-sets are of interest because, given an $r \times s$ matrix $A$ with integer coefficients the following statements are equivalent:

(1) Whenever $\mathscr{F}$ is a finite partition of $N$ there exist $B \in \mathscr{F}$ and $\vec{x} \in B^{s}$ such that $A \vec{x}=\overrightarrow{0}$. (That is, the system $A \vec{x}=\overrightarrow{0}$ is partition regular.)

(2) There exist $m, p, c \in N$ such that, given any $(m, p, c)$-set $B$ there exists $\vec{x} \in B^{s}$ with $A \vec{x}=\overrightarrow{0}$.

Our proof was suggested in the last paragraph of [11, Chapter 8].

The definition of $(m, p, c)$-set which we give below differs from that in 6 and 15] (and for that matter from [1]) in two respects. A trivial difference, for our notational convenience, is to require the coefficient $c$ last rather than first. The other difference is that we restrict the coefficients to $\{1,2, \ldots, p\}$ rather than to $\{-p,-p,+1, \ldots, p-1, p\}$. We shall point out after the definition why this difference is not substantive. The reason for using the different restrictions is that we want to consider the possibility of extending the $(m, p, c)$-set Theorem to arbitrary pre-natural semigroups, in which case $-1 x$ and $-2 x$ may not make sense. (It will turn out not to be possible to adapt our proof, but we want this impossibility to be for substantive reasons.)

5.1 Definition. Let $m, p, c \in N$ and let $\vec{x} \in N^{m}$, then $S(m, p, c, \vec{x})=$ $\left\{c x_{1}\right\} \cup\left\{\sum_{i=1}^{k} \lambda_{i} x_{i}+c x_{k+1}: k \in\{1,2, \ldots, m-1\}\right.$ and for each $i, \lambda_{i} \in\{1,2$, $\ldots, p\}\}$.

We show that any central set in $(N,+)$ contains for each $m, p, c$ some $S(m, p, c, \vec{x})$. If $S^{\prime}(m, p, c, \vec{x})$ is defined as above except that each $\lambda_{i} \in$ $\{-p,-p+1, \ldots, p-1, p\}$ the corresponding result follows from the following simple fact: Given $m, p, c$ and $\vec{x}$, let $p^{*}=(c+1)(1+p)^{m-1}$ and for $i \in\{1,2, \ldots, m\}$ let $y_{i}=\sum_{t=1}^{i}(1+p)^{i-t} x_{t}$. Then $S^{\prime}(m, p, c, \vec{y}) \subseteq$ $S\left(m, p^{*}, c, \vec{x}\right)$. Thus given a central set $A$, one finds $\vec{x}$ with $S\left(m, p^{*}, c, \vec{x}\right)$ $\subseteq A$ and concludes $S^{\prime}(m, p, c, \vec{y}) \subseteq A$. 
5.2 Theorem. Let $A$ be a central set in $(N,+)$ and let $m, p, c \in N$. For each $i \in\{1,2, \ldots, m\}$, there exists a sequence $\left\langle x_{i, n}\right\rangle_{n=1}^{\infty}$ such that for any finite nonempty subset $F$ of $N, S\left(m, p, c, \vec{x}_{F}\right) \subseteq A$, where $\vec{x}_{F}=\left(\sum_{n \in F} x_{1, n}\right.$, $\left.\sum_{n \in F} x_{2, n}, \ldots, \sum_{n \in F} x_{m, n}\right)$.

Proof. Pick a minimal idempotent $q$ with $A \in q$. Note that, as in the proof of Lemma 4.6, $N n \in q$ for each $n$. We prove the theorem by induction on $m$. For $m=1$, since $q+q=q$ and $A \cap N c \in q$, pick by Lemma 4.5, some sequence $\left\langle y_{1, n}\right\rangle_{n=1}^{\infty}$ with $F S\left(\left\langle y_{1, n}\right\rangle_{n=1}^{\infty}\right) \subseteq A \cap N c$. For each $n$ let $x_{1, n}=y_{1, n} / c$. Then given $F, S\left(1, p, c, \vec{x}_{F}\right)=\left\{c \sum_{n \in F} x_{1, n}\right\}=\left\{\sum_{n \in F} y_{1, n}\right\} \subseteq A$.

Now let $m, p, c \in N$ be given. We assume the theorem is valid for $(m, p, c)$ and show it holds for $(m+1, p, c)$. For each $i \in\{1,2, \ldots, m\}$ pick $\left\langle x_{i, n}\right\rangle_{n=1}^{\infty}$ such that $S\left(m, p, c, \vec{x}_{F}\right) \subseteq A$ for each finite nonempty $F \subseteq N$. We apply Theorem 4.11 with $l=p^{m}$. For each $\lambda:\{1,2, \ldots, m\} \rightarrow\{1,2, \ldots, p\}$, and each $n \in N$, define $y_{\lambda, n}=\sum_{i=1}^{m} \lambda_{i} x_{i, n}$. Since $A \cap N c$ is a central set, pick a sequence $\left\langle a_{n}\right\rangle_{n=1}^{\infty}$ in $N$ and a sequence $\left\langle H_{n}\right\rangle_{n=1}^{\infty}$ of pairwise disjoint finite nonempty subsets of $N$ such that whenever $F$ is a finite nonempty subset of $N$ one has $\sum_{n \in F} a_{n} \in A \cap N c$ and, for each $\lambda:\{1,2, \ldots, m\} \rightarrow\{1,2, \ldots, p\}$, $\sum_{n \in F}\left(a_{n}+\sum_{t \in H_{n}} y_{\lambda, t}\right) \in A \cap N c$. For each $i \in\{1,2, \ldots, m\}$ and each $n \in N$ let $z_{i, n}=\sum_{t \in H_{n}} x_{i, t}$ and let $z_{m+1, n}=a_{n} / c$.

Let $F$ be a finite nonempty subset of $N$. We claim $S\left(m+1, p, c, \vec{z}_{F}\right) \subseteq A$. Let $G=\bigcup_{n \in F} H_{n}$. Now

$$
\begin{aligned}
c \cdot \sum_{n \in F} z_{1, n} & =c \cdot \sum_{n \in F} \sum_{t \in H_{n}} x_{1, t} \\
& =c \cdot \sum_{t \in G} x_{1, t} \in S\left(m, p, c, \vec{x}_{G}\right) \subseteq A .
\end{aligned}
$$

Likewise if $k \in\{1,2, \ldots, m-1\}$ and for each $i \in\{1,2, \ldots, k\}, \lambda_{i} \in$ $\{1,2, \ldots, p\}$, then $\sum_{i=1}^{k} \lambda_{i} \cdot \sum_{n \in F} z_{i, n}+c \cdot \sum_{n \in F} z_{k+1, n}=\sum_{i=1}^{k} \lambda_{i} \cdot \sum_{t \in G} x_{i, t}+$ $c \cdot \sum_{t \in G} x_{k+1, t} \in S\left(m, p, c, \vec{x}_{G}\right) \subseteq A$. Finally let

$$
\lambda:\{1,2, \ldots, m\} \rightarrow\{1,2, \ldots, p\}
$$

and consider $d=\sum_{i=1}^{m} \lambda_{i} \cdot \sum_{n \in F} z_{i, n}+c \cdot \sum_{n \in F} z_{m+1, n}$. Then $c \cdot \sum_{n \in F} z_{m+1, n}=$ $\sum_{n \in F} c z_{m+1, n}=\sum_{n \in F} a_{n}$. Also $\sum_{i=1}^{m} \lambda_{i} \cdot \sum_{n \in F} z_{i, n}=\sum_{i=1}^{m} \lambda_{i} \sum_{n \in F} \sum_{t \in H_{n}} x_{i, t}$ $=\sum_{i=1}^{m} \sum_{n \in F} \sum_{t \in H_{n}} \lambda_{i} x_{i, t}=\sum_{n \in F} \sum_{t \in H_{n}} \sum_{i=1}^{m} \lambda_{i} x_{i, t}=\sum_{n \in F} \sum_{t \in H_{n}} y_{\lambda, t}$. Thus $d=\sum_{n \in F}\left(a_{n}+\sum_{t \in H_{n}} y_{\lambda, t}\right) \in A$.

We note that Theorem 5.2 does not generalize to arbitrary pre-natural semigroups. Our definition of $S(m, p, c, \vec{x})$ makes sense in any semigroup. However as a consequence of Theorem 5.3, $\left\{\left\{x^{2}: x \in N\right.\right.$ and $\left.x>1\right\}$, $\left.N \backslash\left\{x^{2}: x \in N\right\}\right\}$ is a partition of $N \backslash\{1\}$ whose only multiplicatively central set is $N \backslash\left\{x^{2}: x \in N\right\}$ which trivially cannot contain the multiplicative version of any $S(m, p, 2, \vec{x})$. 
5.3 Theorem. The set $\left\{x^{2}: x \in N\right.$ and $\left.x>1\right\}$ is not central in $(N \backslash\{1\}, \cdot)$. Proof. Let $A=\left\{x^{2}: x \in N\right.$ and $\left.x>1\right\}$ and suppose that $A$ is central in $(N \backslash\{1\}, \cdot)$. Pick a minimal right ideal $R$ of $\beta(N \backslash\{1\})$ and pick $q \in R$ such that $q \cdot q=q$ and $A \in q$. Let $S=N \backslash\{1\}$. Then $q \cdot \beta S \subseteq R \cdot \beta S \subseteq R$ so $q \cdot \beta S=R$. Since $A \in q=q \cdot q,\{x \in S: A / x \in q\} \in q$ and is hence nonempty. Thus, by Lemma 1.1, there is some finite $F \subseteq S$ such that for all $y \in S,\left(\bigcup_{x \in F} A / x\right) / y \in q$. Pick $n \in N$ with $F \subseteq\{2,3,4, \ldots, n\}$ and pick a prime $p>n$. Now $\left(\bigcup_{x \in F} A / x\right) / p \in q$ and $\left(\bigcup_{x \in F} A / x\right) / p^{2} \in q$ so pick $z \in\left(\left(\bigcup_{x \in F} A / x\right) / p\right) \cap\left(\left(\bigcup_{x \in F} A / x\right) / p^{2}\right)$. Then for some $x$ and $y$ in $F$ $z p x \in A$ and $z p^{2} y \in A$. Then $x$ and $y$ have no factors of $p$. Thus, since $z p x \in A, z$ has an odd number of factors of $p$ while since $z p^{2} y \in A, z$ has an even number of factors of $p$, a contradiction.

One of the major combinatorial advantages of working with $\beta N$ is the existence of two interacting operations. We see here that this interaction applies also to central sets.

5.4 Theorem. Let $M=\operatorname{cl}\{p: p$ is a minimal idempotent in $(\beta N,+)\}$. Then $M$ is a right ideal of $(\beta N, \cdot)$.

Proof. Let $q \in M$, let $r \in \beta N$, and let $A \in q \cdot r$. We need to show there is some minimal idempotent of $(\beta N,+)$ in $\bar{A}$. Now $\{x \in N: A / x \in q\} \in r$ so pick $x \in N$ with $A / x \in q$. Since $q \in M$, pick a minimal idempotent $p$ of $(\beta N,+)$ with $A / x \in p$. Then $A \in p \cdot x$. Also by Lemma $1.2, p \cdot x+p \cdot x=p \cdot x$.

To complete the proof we need to show that $p \cdot x$ is minimal, that is that $p \cdot x$ is in some minimal right ideal of $(\beta N,+)$. Since $p \cdot x=p \cdot x+p \cdot x \in p \cdot x+\beta N$, it suffices to show $p \cdot x+\beta N$ is a minimal right ideal.

Now $p$ is in some minimal right ideal $R$ and $p+\beta N \subseteq R+\beta N \subseteq R$ so $p+\beta N=R$. To see that $p \cdot x+\beta N$ is a minimal right ideal we use Lemma 1.1. Let $B \subseteq N$ and assume there is some $n \in N$ with $B-n \in p \cdot x$. We need to produce a finite $F \subseteq N$ such that for each $y \in N,\left(\bigcup_{z \in F} B-z\right)-y \in p \cdot x$.

Pick $n \in N$ with $B-n \in p \cdot x$ and pick $i \in\{0,1, \ldots, x-1\}$ such that $n+i \in N x$. Then $(B+i)-(n+i)=B-n \in p \cdot x$ so $(B+i) / x-(n+i) / x \in p$. (Recall that $(B+i) / x=\{y \in N: y x \in B+i\}=\{z / x: z \in(B+i) \cap N x\}$.)

Let $C=(B+i) / x$. Since $C-(n+i) / x \in p$, pick by Lemma 1.1 , finite $F \subseteq N$ such that for all $y \in N,\left(\bigcup_{z \in F} C-z\right)-y \in p$. Pick $k \in N$ with $F \subseteq\{1,2, \ldots, k\}$ and let $G=\{1,2, \ldots,(k+1) \cdot x\}$. We claim that for each $y \in N,\left(\bigcup_{z \in G} B-z\right)-y \in p \cdot x$. To this end, let $y \in N$ and pick $a \in N$ such that $(a-1) x \leq y<a x$. Then $\left(\bigcup_{z \in F} C-z\right)-a \in p$ and $\left(\bigcup_{z \in F} C-z\right)-a=\bigcup_{z \in F} C-(a+z)$ so pick $t \in F$ with $C-(a+t) \in p$. Then $C x-(a+t) x \in p \cdot x$. Now $C x-(a+t) x \subseteq B+i-a x-t x$ so $B+i-a x-t x \in p \cdot x$. Pick $s \in\{1,2, \ldots, x\}$ such that $a x=y+s$. Then $B+i-a x-t x=(B-(t x-i+s))-y \subseteq\left(\bigcup_{z \in G} B-z\right)-y$ so $\left(\bigcup_{z \in G} B-z\right)-y \in$ $p \cdot x$ as required. 
5.5 Corollary. Let $\mathscr{F}$ be a finite partition of $N$. There is some $A \in \mathscr{F}$ which is central in $(N,+)$ and in $(N, \cdot)$. In particular, there is one cell of $\mathscr{F}$ satisfying both the multiplicative and additive versions of Theorem 4.11.

Proof. Let $M=\operatorname{cl}\{p: p$ is a minimal idempotent in $(\beta N,+)\}$. By Theorem $5.4 M$ is a right ideal in $(\beta N, \cdot)$ so, by Zorn's Lemma pick a minimal right ideal $R \subseteq M$. By [8, Corollary 2.10], pick an idempotent $q=q \cdot q$ in $R$. Pick $A \in \mathscr{F}$ with $A \in q$. Since $q$ is a minimal idempotent in $(\beta N, \cdot) A$ is central in $(N, \cdot)$. Since $A \in q$ and $q \in M$, there is some minimal idempotent $p$ in $(\beta N,+)$ with $A \in p$. Thus $A$ is central in $(N,+)$.

In [1] we established the existence of a "combinatorially large"ultrafilter in $\beta N$, deducing that some cell of any finite partition of $N$ satisfies a long list of combinatorial statements. We show here, in Theorem 5.6, that we may choose a combinatorially large ultrafilter such that each member of $q$ is central in $(N,+)$ and in $(N, \cdot)$. We thus add both the additive and multiplicative versions of Theorem 4.11 to our list of combinatorial conclusions about one cell of a partition.

It would take us too far afield to include all of the definitions from [1]. The reader who wants to follow the proof of Theorem 5.6 will need to have a copy of [1] available.

5.6 Theorem. There is a combinatorially large ultrafilter $q$ such that $q$ is a minimal idempotent in $(\beta N, \cdot)$ and each member of $q$ is central in $(N,+)$.

Proof. Let $M$ be as in Theorem 5.4 and let $M P C, L F S, \Delta, \Delta_{1}, \Delta_{m}$ and $U$ be as defined in [1]. We need to show there is some $q$, a minimal idempotent in $(\beta N, \cdot)$ with $q \in M P C \cap L F S \cap \Delta_{1} \cap \Delta_{m} \cap M$.

Now $\Delta$ is a right ideal of $(\beta N,+)$ [20, Theorem 10.8] so pick a minimal right ideal $R \subseteq \Delta$ and pick an idempotent $r \in R$. By Theorem 5.2 and the remarks preceding Theorem 5.2, $r \in U$. By [1, Lemma 2.4] $r \in M P C$. Since $r \in R$ and $r+r=r, r \in M$. Thus $r \in M \cap M P C$. Since $M$ is a right ideal of $(\beta N, \cdot)$ (by Theorem 5.4) and MPC is a right ideal of $(\beta N, \cdot)$ (by [1, Lemma 2.4]), we have $M \cap M P C$ is a right ideal of $(\beta N, \cdot)$ so $r \cdot r \in M \cap M P C$. By [1, Lemma 2.13] $r \cdot r \in L F S$ so $M \cap M P C \cap L F S \neq \varnothing$ so, by [1, Lemma 2.14] $M \cap M P C \cap L F S$ is a right ideal of $(\beta N, \cdot)$. Pick a minimal right ideal $J$ of $(\beta N, \cdot)$ with $J \subset M \cap M P C \cap L F S$ and pick $q \in J$ such that $q \cdot q=q$. We claim $q$ is as required. Immediately $q$ is a minimal idempotent in $(\beta N, \cdot)$ and $q \in M \cap M P C \cap L F S$. To show $q \in \Delta_{1} \cap \Delta_{m}$ it suffices to show $M \subseteq \Delta_{1}$ and $J \subseteq \Delta_{m}$. By [1, Lemmas 2.6 and 2.8] $\Delta_{1}$ and $\Delta_{m}$ are ideals of $(\beta N,+)$ and $(\beta N, \cdot)$ respectively while $J$ is a minimal right ideal of $(\beta N, \cdot)$ and $M$ is contained in the closure of the minimal ideal of $(\beta N,+)$.

We conclude this section by showing in Corollary 5.9 that central sets are common in $N$; in fact sets which are central both multiplicatively and additively are common. 
5.7 Lemma. Each right ideal in $(\beta N, \cdot)$ contains $2^{c}$ idempotents, where $c$ is the cardinality of the continuum.

Proof. It is well known (see for example [22, Corollary 2.6]) that each right ideal in $(\beta \omega,+)$ has $2^{c}$ idempotents.

Let $R$ be a right ideal in $(\beta N, \cdot)$. We may presume $R$ is a minimal right ideal (since $R$ contains a minimal right ideal). Define $\varphi: N \rightarrow \omega$ by $\varphi(x)$ is the length of the prime factorization of $x$. Then $\varphi$ is a homomorphism from $(N, \cdot)$ onto $(\omega,+)$. By a theorem of Milnes [24] (or see [22]), the continuous extension $\varphi^{\beta}: \beta N \rightarrow \beta \omega$ is a homomorphism from $(\beta N, \cdot)$ onto $(\beta \omega,+)$. Consequently $P=\varphi^{\beta}[R]$ is a right ideal of $(\beta \omega,+)$ and hence contains $2^{c}$ idempotents. It thus suffices to show that if $q \in P$ and $q+q=q$, then $\left(\varphi^{\beta}\right)^{-1}[\{q\}] \cap R$ contains an idempotent. Since $q \in \varphi^{\beta}[R]$, this intersection is nonempty and is thus a compact left topological semigroup which therefore contains an idempotent as required.

5.8 Theorem. There are $2^{c}$ combinatorially large ultrafilters which are minimal idempotents in $(\beta N, \cdot)$ and every member of which is central in $(N,+)$.

Proof. In the proof of Theorem 5.6, we obtain a right ideal $J$ of $(\beta N, \cdot)$ such that every idempotent of $J$ satisfies the conclusions of this theorem. By Lemma 5.7, there are $2^{c}$ such idempotents.

Our proof of Corollary 5.9 uses a standard topological argument.

5.9 Corollary. There is a partition of $N$ into infinitely many cells, each one of which is central in $(N,+)$ and is a member of a combinatorially large ultrafilter which is a minimal idempotent in $(\beta N, \cdot)$.

Proof. By Theorem 5.8, pick a sequence $\left\langle q_{n}\right\rangle_{n=1}^{\infty}$ of distinct combinatorially large ultrafilters which are minimal idempotents in $(\beta N, \cdot)$ and each member of which is central in $(N,+)$. Pick $B_{1,0} \in q_{1}$ and $B_{1,1} \in q_{2}$ with $B_{1,0} \cap B_{1,1}=$ $\varnothing$. Pick $\sigma(1) \in\{0,1\}$ so that $C_{1}=\left\{k \in N: q_{k} \notin B_{1, \sigma(1)}\right\}$ is infinite. Let $p_{1}=q_{1}$ or $p_{1}=q_{2}$ depending on whether $\sigma(1)=0$ or $\sigma(1)=1$.

Inductively, given infinite $C_{n-1}=\left\{k \in N: q_{k} \notin \bigcup_{i=1}^{n-1} B_{i, \sigma(i)}\right\}$ pick distinct $s, t \in C_{n-1}$ and pick $B_{n, 0} \in q_{s}$ and $B_{n, 1} \in q_{t}$ with $B_{n, 0} \cap B_{n, 1}=\varnothing$. Pick $\sigma(n) \in\{0,1\}$ such that $C_{n}=\left\{k \in C_{n-1}: q_{k} \notin B_{n, \sigma(n)}\right\}$ is infinite. Note that $C_{n}=\left\{k \in N: q_{k} \notin \bigcup_{i=1}^{n} B_{i, \sigma(i)}\right\}$. Let $p_{n}=q_{s}$ if $\sigma(n)=0$ and let $p_{n}=q_{t}$ if $\sigma(n)=1$.

For each $n>1$, let $A_{n}=B_{n, \sigma(n)} \backslash \bigcup_{i=1}^{n-1} B_{i, \sigma(i)}$ and let $A_{1}=N \backslash \bigcup_{n=2}^{\infty} A_{n}$. Observe that $B_{1, \sigma(1)} \subseteq A_{1}$. Then each $A_{n} \in p_{n}$ hence is as required.

\section{THE NOTIONS OF "CENTRAL"}

We were motivated to call members of minimal idempotents "central" when we found out we could prove Theorem 4.11 which is, as we have seen a generalization of [11, Proposition 8.21], a result about "central" sets in $N$. We 
establish here that the similar results obtained are not a coincidence. In fact, for a countable semigroup $S$, the notions coincide. ${ }^{1}$ (One of the two implications involved is due to $\mathrm{B}$. Weiss.)

We are grateful to Dennis Davenport who pointed out that we need not restrict ourself to commutative semigroups.

We first recall some definitions (following [11] in generalizing the notion to arbitrary semigroups).

6.1 Definition. A dynamical system is a pair $\left(X,\left\langle T_{s}\right\rangle_{s \in S}\right)$ where $(X, d)$ is a compact metric space, $(S,+)$ is a semigroup, each $T_{s}$ is a continuous function from $X$ to $X$, and for $s, t \in S, T_{s} \circ T_{t}=T_{t+s}$.

6.2 Definition. Let $\left(X,\left\langle T_{s}\right\rangle_{s \in S}\right)$ be a dynamical system.

(a) Points $x$ and $y$ in $X$ are proximal if and only if there is a sequence $\langle s(k)\rangle_{k=1}^{\infty}$ in $S$ such that $\lim _{k \rightarrow \infty} d\left(T_{s(k)}(x), T_{s(k)}(y)\right)=0$.

(b) A subset $D$ of $S$ is syndetic if and only if there is a finite subset $G$ of $S$ such that for each $s \in S$ there exists $z \in G$ with $s+z \in D$.

(c) A point $y$ in $X$ is uniformly recurrent if and only if for each neighborhood $U$ of $y,\left\{s \in S: T_{s}(y) \in U\right\}$ is syndetic.

In [11], the definition of "central" applied only to subsets of $N$. However, a verbatim application is meaningful in any semigroup. We denote this notion here by "*-central."

6.3 Definition. Let $(S,+)$ be a semigroup. A set $A \subseteq S$ is *-central if and only if there exist $X$ and $\left\langle T_{s}\right\rangle_{s \in S}$ such that $\left(X,\left\langle T_{s}\right\rangle_{s \in S}\right)$ is a dynamical system and there exist $x, y \in X$ and a neighborhood $U$ of $y$ such that $x$ and $y$ are proximal, $y$ is uniformly recurrent, and $A=\left\{s \in S: T_{s}(x) \in U\right\}$.

In showing that ${ }^{*}$-central sets are central, the main tool is the notion of enveloping semigroup developed by Ellis [7 and 9]. Given a dynamical system $\left(X,\left\langle T_{s}\right\rangle_{s \in S}\right)$ one takes the set ${ }^{X} X$ of all functions from $X$ to $X$ under composition with the product topology and lets $E=\operatorname{cl}\left\{T_{s}: s \in S\right\}$. Then $E$ is a compact right topological semigroup. That is, for each $f \in E$, the function $\rho_{f}$ defined by $\rho_{f}(g)=g \circ f$ is continuous. Further, if $f$ is continuous (in particular if $f=T_{s}$ for some $s \in S$ ) then $\lambda_{f}$ is continuous. (See for example $[11$, p. 159].)

Our first lemma is essentially [8, Lemma 5.15] except that we are not working with a group.

6.4 Lemma. Let $\left(X,\left\langle T_{s}\right\rangle_{s \in S}\right)$ be a dynamical system, let $E$ be its enveloping semigroup, and let $x$ and $y$ be proximal in $X$. There is a minimal left ideal $L$ of $E$ such that $f(x)=f(y)$ whenever $f \in L$.

Proof. Let $L=\{f \in E: f(x)=f(y)\}$. It suffices to show that $L \neq \varnothing$. For then $L$ is trivially a left ideal and hence, by an easy application of Zorn's lemma

\footnotetext{
${ }^{1}$ This result was anticipated by $\mathrm{S}$. Glasner who showed essentially the same thing for abelian groups in Proposition 4.6 of Canad. J. Math. 32 (1980), 993-1007.
} 
(using the fact that $E$ is right topological) $L$ contains a minimal left ideal.

Pick a sequence $\langle s(k)\rangle_{k=1}^{\infty} \in S$ such that $\lim _{k \rightarrow \infty} d\left(T_{s(k)}(x), T_{s(k)}(y)\right)=0$ and let $f$ be a cluster point of $\left\langle T_{s(k)}\right\rangle_{k=1}^{\infty}$ in $E$. Suppose that $f(x) \neq f(y)$ and let $\varepsilon=d(f(x), f(y)) / 3$. Pick $m$ such that for all $k>m$

$$
d\left(T_{s(k)}(x), T_{s(k)}(y)\right)<\varepsilon .
$$

Let $V=\pi_{x}^{-1}[N(f(x), \varepsilon)] \cap \pi_{y}^{-1}[N(f(y), \varepsilon)]$. Then $V$ is a neighborhood of $f$ in ${ }^{X} X$ so pick $k>m$ such that $T_{s(k)} \in V$. Then $d\left(T_{s(k)}(x), T_{s(k)}(y)\right)<\varepsilon$, $d\left(T_{s(k)}(x), f(x)\right)<\varepsilon$, and $d\left(T_{s(k)}(y), f(y)\right)<\varepsilon$ so we obtain a contradiction.

6.5 Lemma. Let $\left(X,\left\langle T_{s}\right\rangle_{s \in S}\right)$ be a dynamical system and let $E$ be its enveloping semigroup. Let $L$ be a left ideal of $E$ and let $D$ be a syndetic subset of $S$. Then $L \cap \operatorname{cl}\left\{T_{s}: s \in D\right\} \neq \varnothing$.

Proof. Since $D$ is syndetic, pick a finite subset $G$ of $S$ such that for each $s \in S$ there exists $z \in G$ with $s+z \in D$. Pick $f \in L$. We claim there is some $z \in G$ with $T_{z} \circ f \in \operatorname{cl}\left\{T_{s}: s \in D\right\}$. Since $L$ is a left ideal this will suffice.

Suppose instead that for each $z \in G$ there is a basic neighborhood of $T_{z} \circ f$ missing $\left\{T_{s}: s \in D\right\}$. Pick for each $z \in G$ a finite subset $F_{z}$ of $X$ and an $\varepsilon_{z}>0$ such that $\left\{T_{s}: s \in D\right\} \cap \bigcap_{x \in F_{z}} \pi_{z}^{-1}\left[N\left(T_{z}(f(x)), \varepsilon_{z}\right)\right]=\varnothing$. Now each $T_{z}$ is continuous so, since $X$ is compact, is uniformly continuous so pick $\delta_{z}>0$ such that $d\left(T_{z}(x), T_{z}(y)\right)<\varepsilon_{z}$ whenever $d(x, y)<\delta_{z}$. Let $F=\bigcup_{z \in G} F_{z}$ and let $\delta=\min \left\{\delta_{z}: z \in G\right\}$. Let $W=\bigcap_{x \in F} \pi_{x}^{-1}[N(f(x), \delta)]$. Since $W$ is a neighborhood of $f$ and $f \in L$ and $L \subseteq E=\operatorname{cl}\left\{T_{s}: s \in S\right\}$, pick $s \in S$ with $T_{s} \in W$. Pick $z \in G$ with $s+z \in D$. Now $T_{s} \in W$ so given $x \in F_{z}, d\left(T_{s}(x), f(x)\right)<\delta \leq \delta_{z}$ and hence $d\left(T_{z}\left(T_{s}(x)\right), T_{z}(f(x))\right)<\varepsilon_{z}$. Thus $T_{s+z} \in \bigcap_{x \in F_{z}} \pi_{x}^{-1}\left[N\left(T_{z}(f(x)), \varepsilon_{z}\right)\right]$ while $s+z \in D$. Thus we have a contradiction.

6.6 Lemma. Let $\left(X,\left\langle T_{s}\right\rangle_{s \in S}\right)$ be a dynamical system and let $E$ be its enveloping semigroup. Let $y$ be uniformly recurrent in $X$ and let $L$ be a left ideal of $E$. There exists $f \in L$ with $f \circ f=f$ and $f(y)=y$.

Proof. We may presume $L$ is compact since, if $f \in L, E \circ f=\rho_{f}[E] \subseteq L$ and is compact. Thus it suffices to show there exists $f \in L$ with $f(y)=y$. For then $\{f \in L: f(y)=y\}=L \cap \pi_{y}^{-1}[\{y\}]$ is a compact subsemigroup which then contains an idempotent.

Suppose instead each $f \in L$ has $f(y) \neq y$ and let $\varepsilon_{f}=d(f(y), y) / 2$ and let $V_{f}=\pi_{y}^{-1}\left[N\left(f(y), \varepsilon_{f}\right)\right]$. Then $\left\{V_{f}: f \in L\right\}$ is an open cover of $L$ so pick finite $F \subseteq L$ with $L \subseteq \bigcup_{f \in F} V_{f}$. Let $\varepsilon=\min \left\{\varepsilon_{f}: f \in F\right\}$ and let $D=\left\{s \in S: T_{s}(y) \in N(y, \varepsilon)\right\}$. Since $y$ is uniformly recurrent, $D$ is syndetic. Pick by Lemma 6.5, $g \in L \cap \operatorname{cl}\left\{T_{s}: s \in D\right\}$. Pick $f \in F$ such that $g \in V_{f}$ and, since $V_{f}$ is a neighborhood of $g$, pick $s \in D$ with $T_{s} \in V_{f}$. Then 
$d\left(f(y), T_{s}(y)\right)<\varepsilon_{f}$ and, since $s \in D, d\left(T_{s}(y), y\right)<\varepsilon \leq \varepsilon_{f}$. But then $d(f(y), y)<2 \varepsilon_{f}$, a contradiction.

6.7 Lemma. Let $\left(X,\left\langle T_{s}\right\rangle_{s \in S}\right)$ be a dynamical system and let $E$ be its enveloping semigroup. Define $\varphi: S \rightarrow E$ by $\varphi(s)=T_{s}$ and let $\varphi^{\beta}$ be the continuous extension of $\varphi$ to $\beta S$. If $f$ is an idempotent in a minimal left ideal of $E$, then there is a minimal idempotent $p \in \beta S$ with $\varphi^{\beta}(p)=f$.

Proof. Note that $\varphi^{\beta}[\beta S]$ is compact so that $\varphi^{\beta}[\beta S]=E$. We now establish that $\varphi^{\beta}$ is an antihomomorphism. To this end, let $p, q \in \beta S$ and suppose that $\varphi^{\beta}(p+q) \neq \varphi^{\beta}(q) \circ \varphi^{\beta}(p)$. Pick $U$ and $V$ disjoint open neighborhoods of $\varphi^{\beta}(p+q)$ and $\varphi^{\beta}(q) \circ \varphi^{\beta}(p)$ respectively. Pick $A \in p+q$ such that $\varphi^{\beta}[\bar{A}] \subseteq U$ and let $B=\{s \in S: A-s \in p\}$, so that $B \in q$.

Pick $W$ a neighborhood of $\varphi^{\beta}(q)$ with $\rho_{\varphi^{\beta}(p)}[W] \subseteq V$. Pick $C \in q$ with $\varphi^{\beta}[\bar{C}] \subseteq W$. Pick $s \in B \cap C$. Since $s \in C, T_{s} \in W$ so $T_{s} \circ \varphi^{\beta}(p) \in V$. Since $\lambda_{T_{s}}$ is continuous, pick a neighborhood $M$ of $\varphi^{\beta}(p)$ with $\lambda_{T_{s}}[M] \subseteq V$. Pick $D \in p$ with $\varphi^{\beta}[\bar{D}] \subseteq M$. Since $S \in B, A-s \in p$ so pick $t \in A-s \cap D$. Since $t \in D, T_{t} \in M$ so $T_{s} \circ T_{t} \in V$. Since $t+s \in A, T_{t+s} \in U$ and we have a contradiction.

Now pick a minimal left ideal $L$ of $E$ with $f \in L$. Then $\left(\varphi^{\beta}\right)^{-1}[L]$ is a right ideal of $\beta S$ so pick a minimal right ideal $R$ of $\beta S$ with $R \subseteq\left(\varphi^{\beta}\right)^{-1}[L]$. Then $\varphi^{\beta}[R]$ is a left ideal of $E, \varphi^{\beta}[R] \subseteq L$, and $L$ is minimal so $\varphi^{\beta}[R]=L$. Consequently $\left(\varphi^{\beta}\right)^{-1}[\{f\}] \cap R$ is a (nonempty) compact subsemigroup of $\beta S$ so pick an idempotent $p \in\left(\varphi^{\beta}\right)^{-1}[\{f\}] \cap R$.

6.8 Theorem. Let $\left(X,\left\langle T_{s}\right\rangle_{s \in S}\right)$ be a dynamical system, let $x$ and $y$ be proximal in $X$ with $y$ uniformly recurrent. There is a minimal idempotent $p \in \beta S$ such that, whenever $U$ is a neighborhood of $y,\left\{s \in S: T_{s}(x) \in U\right\} \in p$. In particular, each *-central set in $S$ is central in $S$.

Proof. Let $E$ be the enveloping semigroup of $\left(X,\left\langle T_{s}\right\rangle_{s \in S}\right)$ and define $\varphi: S \rightarrow$ $E$ by $\varphi(s)=T_{s}$. Pick by Lemma 6.4 a minimal left ideal $L$ of $E$ such that $f(x)=f(y)$ whenever $f \in L$. Pick by Lemma 6.6 an idempotent $f \in L$ with $f(y)=y$. Pick by Lemma 6.7 a minimal idempotent $p \in \beta S$ with $\varphi^{\beta}(p)=f$.

Let $U$ be a neighborhood of $y$ and let $A=\left\{s \in S: T_{s}(x) \in U\right\}$. Now $\varphi^{\beta}(p)(x)=f(x)=f(y)=y$ and $y \in U$ so $\pi_{x}^{-1}[U]$ is a neighborhood of $\varphi^{\beta}(p)$. Pick $B \in p$ such that $\varphi^{\beta}[\bar{B}] \subseteq \pi_{x}^{-1}[U]$. We claim that $B \subseteq A$ so that $A \in p$. Let $s \in B$. Then $T_{s} \in \pi_{x}^{-1}[U]$ so $s \in A$.

The notion of "central" is closed under supersets while it is not obvious that the corresponding statement holds for "*-central". Accordingly we asked (with $S$ as the natural numbers under addition): "Given a central set $A$ in $S$ must there be a *-central set $B$ with $B \subseteq A$ ?" This question was answered in the affirmative by $B$. Weiss. We are grateful to him for permission to present 
his elegant proof (in Theorem 6.11). The proof uses the notion of " $p$-lim" (Definition 2.2).

6.9 Lemma. Let $\left(X,\left\langle T_{s}\right\rangle_{s \in S}\right)$ be a dynamical system. Let $R$ be a minimal right ideal of $\beta S$ and let $p \in R$. If $y \in X$ and $p-\lim _{s \in S} T_{s}(y)=y$, then $y$ is uniformly recurrent.

Proof. Let $\varepsilon>0$ be given and let $A=\left\{s \in S: d\left(T_{s}(y), y\right)<\varepsilon\right\}$. We need to show that $A$ is syndetic. Let $B=\left\{s \in S: d\left(T_{s}(y), y\right)<\varepsilon / 2\right\}$. Since $p-\lim _{s \in S} T_{s}(y)=y$, we have $B \in p$.

Let $C=\{t \in S: B-t \in p\}$. We claim that $C$ is syndetic. Since $p+\beta S \subseteq$ $R+\beta S \subseteq R$ we have $p+\beta S=R$. Thus $p+q=p$ for some $q \in \beta N$. Since $B \in p, C \in q$ so $C \neq \varnothing$. Pick by Lemma 1.1 a finite $F \subseteq S$ such that for all $y \in S,\left(\bigcup_{x \in F} B-x\right)-y \in p$. Then, given $y \in S, \bigcup_{x \in F} B-(y+x) \in p$ so there is some $x \in F$ with $B-(y+x) \in p$ (so that $y+x \in C$ as required).

We now claim that $C \subseteq A$, so that $A$ is syndetic. To this end let $t \in C$. Since $T_{t}$ is continuous and $X$ is compact, $T_{t}$ is uniformly continuous. Pick $\delta>0$ such that $d\left(T_{t}(x), T_{t}(z)\right)<\varepsilon / 2$ whenever $x, z \in X$ with $d(x, z)<\delta$. Let $D=\left\{s \in S: d\left(T_{s}(y), y\right)<\delta\right\}$. Since $p-\lim _{s \in S} T_{s}(y)=y$, we have $D \in p$. Pick $s \in D \cap(B-t)$. Since $s \in D, d\left(T_{s}(y), y\right)<\delta$ so

$$
d\left(T_{t}\left(T_{s}(y)\right), T_{t}(y)\right)<\varepsilon / 2 .
$$

Since $s+t \in B, d\left(T_{s+t}(y), y\right)<\varepsilon / 2$. Since $T_{t} \circ T_{s}=T_{s+t}, d\left(T_{t}(y), y\right)<\varepsilon$ as required.

6.10 Lemma. Let $X$ be a Hausdorff space, let $S$ be a semigroup, let $p, q \in$ $\beta S$ and let $\left\langle x_{s}\right\rangle_{s \in S}$ be an indexed family in $X$. Assume that for each $t \in$ $S, q-\lim _{s \in S} x_{s+t}$ exists and that $p-\lim _{t \in S}\left(q-\lim _{s \in S} x_{s+t}\right)$ exists. Then $(q+p)-$ $\lim _{r \in S} x_{r}=p-\lim _{t \in S}\left(q-\lim _{s \in S} x_{s+t}\right)$.

Proof. For each $t \in S$ let $y_{t}=q-\lim _{s \in S} x_{s+t}$ and let $z=p-\lim _{t+s} y_{t}$. To see that $z=(q+p)-\lim _{r \in S} x_{r}$, let $U$ be an open neighborhood of $z$ and let $A=\left\{r \in S: x_{r} \in U\right\}$. We show that $A \in q+p$. Let $B=\left\{t \in S: y_{t} \in U\right\}$. Then $B \in p$. We claim that $B \subseteq\{t \in S: A-t \in q\}$. Let $t \in B$ and let $C=\left\{s \in S: x_{s+t} \in U\right\}$. Since $y_{t} \in U, C \in q$. To complete the proof we show $C \subseteq A-t$. Let $s \in C$. Then $x_{s+t} \in U$ so $s+t \in A$ as required.

6.11 Theorem (Weiss). Let $S$ be a countable semigroup and let $A$ be central in $S$. Then $A$ is *-central in $S$.

Proof. Let $S^{*}=S \cup\{0\}$, where 0 is a new two-sided identity adjointed to $S$. Let $X$ be the set of functions from $S^{*}$ to $\{0,1\}$ with the product topology. Enumerate $S^{*}$ faithfully as $\left\{a_{n}: n \in N\right\}$ and for $x \neq y$ in $X$ define $d(x, y)=$ $1 / t$ where $t=\min \left\{n: x\left(a_{n}\right) \neq y\left(a_{n}\right)\right\}$. Then $d$ is a metric on $X$ and the metric topology and the product topology agree.

For each $s \in S$, define $T_{s}: X \rightarrow X$ by $T_{s}(x)(t)=x(s+t)$. Then each $T_{s}$ is continuous. (Let $s \in S$ and consider a subbasic open set $\pi_{t}^{-1}[\{\alpha\}]$ where $t \in S^{*}$ 
and $\alpha \in\{0,1\}$. Then $T_{s}^{-1}\left[\pi_{t}^{-1}[\{\alpha\}]\right]=\pi_{s+t}^{-1}[\{\alpha\}]$.) Also, given $r, s \in S$ and $t \in S^{*}$ and $x \in X$ we have $\left(T_{r} \circ T_{s}\right)(x)(t)=T_{r}\left(T_{s}(x)\right)(t)=T_{s}(x)(r+t)=$ $x(s+r+t)=T_{s+r}(x)(t)$. Thus $\left(X,\left\langle T_{s}\right\rangle_{s \in S}\right)$ is a dynamical system.

Let $x=\chi_{A}$, the characteristic function of $A$. Pick a minimal right ideal $R$ of $\beta S$ and an idempotent $p \in R$ with $A \in p$. Let $y=p-\lim _{r \in S} T_{r}(x)$. As is easy to verify, given $t \in S$ and $\left\langle z_{s}\right\rangle_{s \in S}$ in $X, p-\lim _{s \in S} T_{t}\left(z_{s}\right)=$ $T_{t}\left(p-\lim _{s \in S} z_{s}\right)$. Since $p=p+p$ we have, using Lemma 6.10,

$$
\begin{aligned}
y & =(p+p) \lim _{r \in S} T_{r}(x) \\
& =p-\lim _{t \in S}\left(p-\lim _{s \in S} T_{s+t}(x)\right) \\
& =p-\lim _{t \in S}\left(p-\lim _{s \in S} T_{t}\left(T_{s}(x)\right)\right) \\
& =p-\lim _{t \in S} T_{t}\left(p-\lim _{s \in S T_{s}}(x)\right) \\
& =p-\lim _{t \in S} T_{t}(y) .
\end{aligned}
$$

Thus by Lemma 6.9, $y$ is uniformly recurrent. Since $p$ - $\lim _{s \in S} T_{s}(x)=$ $p$ - $\lim _{s \in S} T_{s}(y)$, we easily get that $x$ and $y$ are proximal.

Let $U=\pi_{0}^{-1}[\{y(0)\}]=\{z \in X: z(0)=y(0)\}$. We show that $A=\{s \in$ $\left.S: T_{s}(x) \in U\right\}$ so that $A$ is ${ }^{*}$-central. Since $y=p-\lim _{s \in S} T_{s}(x)$, we have that $\left\{s \in S: T_{s}(x) \in U\right\} \in p$ so we may pick $t \in A$ with $T_{t}(x) \in U$. Then $y(0)=x(t)=1$, since $t \in A$. Thus $U=\{z \in X: z(0)=1\}$. But then, given $s \in S$,

$$
\begin{aligned}
T_{s}(x) \in U & \Leftrightarrow T_{s}(x)(0)=1 \\
& \Leftrightarrow x(s)=1 \\
& \Leftrightarrow s \in A .
\end{aligned}
$$

6.12 Corollary. Let $S$ be a countable semigroup and let $A \subseteq S$. Then $A$ is central in $S$ if and only if $A$ is *-central in $S$.

As we remarked earlier, it is not obvious that the notion of ${ }^{*}$-central is closed under supersets. It is, however, true, at least for countable semigroups.

6.13 Corollary. Let $S$ be a countable semigroup and let $A \subseteq B \subseteq S$. If $A$ is *-central, then $B$ is *-central.

Proof. $A$ is *-central so by Theorem $6.8, A$ is central. Pick a minimal idempotent $p$ in $\beta S$ with $A \in p$. Then $B \in p$ so $B$ is central so by Theorem 6.11 , $B$ is *-central.

We also see that (for countable semigroups) in the definition of *-central, it suffices to work with one concrete dynamical system.

6.14 Corollary. Let $S$ be a countable semigroup and let $X$ and $\left\langle T_{s}\right\rangle_{s \in S}$ be as in the proof of Theorem 6.11. Let $A \subseteq S$. Then $A$ is *-central if and only if 
there exist $x, y \in X$ with $y$ uniformly recurrent and $x$ proximal to $y$, and $a$ neighborhood $U$ of $Y$ such that $A=\left\{s \in S: T_{s}(x) \in U\right\}$.

Proof. The sufficiency is trivial so assume $A$ is *-central. By Theorem $6.8 A$ is central so the proof of Theorem 6.11 applies.

The semigroup $S$ must be countable in order for the space $X$ produced in the proof of Theorem 6.11 to be metric. In light of Theorem 6.8 , the following question is thus natural.

6.15 Question. Let $S$ be an uncountable semigroup. Must every central set in $S$ contain a *-central set?

Note that the correspondences established in Theorems 6.8 and 6.11 are of a different nature. Central sets are produced by minimal idempotents while *-central sets are produced by pairs $(x, y)$. In Theorem 6.8 it was shown that given a pair $(x, y)$ there is a minimal idempotent $p$ such that all *-central sets produced by $(x, y)$ are central produced by $p$. On the other hand, in Theorem 6.11 , the pair $(x, y)$ depends both on the central set and the idempotent. We conclude by showing that this distinction is necessary.

6.16 Theorem. Let $R$ be a minimal right ideal of $(\beta N,+)$ and let $p \in R$ with $p+p=p$. There do not exist a dynamical system $(X, T)$ and $x, y \in X$ such that

(1) $y$ is uniformly recurrent.

(2) $x$ and $y$ are proximal, and

(3) for each $A \in p$ there exists a neighborhood $U$ of $y$ with $\left\{n \in N: T^{n}(x)\right.$ $\in U\} \subseteq A$.

Proof. Suppose we have such $(X, T), x$, and $y$. For each $m \in N$ let $B_{m}=$ $\left\{n \in N: d\left(T^{n}(x), y\right)<1 / m\right\}$. Then each $B_{m}$ is infinite. (In the context of the current paper one can see this by observing that $B_{m}$ is *-central, hence central.) For each $m$ pick $a_{m} \in B_{m} \backslash\left(\left\{a_{k}: k<m\right\} \cup\left\{b_{k}: k<m\right\}\right)$ and $b_{m} \in$ $B_{m} \backslash\left(\left\{a_{k}: k \leq m\right\} \cup\left\{b_{k}: k<m\right\}\right)$. Then $\left\{a_{m}: m \in N\right\} \cap\left\{b_{m}: m \in N\right\}=\varnothing$ so, without loss of generality $\left\{a_{m}: m \in N\right\} \notin p$. Let $A=N \backslash\left\{a_{m}: m \in N\right\}$. Pick $U$, a neighborhood of $y$, with $A \supseteq\left\{n \in N: T^{n}(x) \in U\right\}$. Pick $m \in N$ with $\{z \in X: d(z, y)<1 / m\} \subseteq U$. Then $d\left(T^{a_{m}}(x), y\right)<1 / m$ so $T^{a_{m}}(x) \in U$ so $a_{m} \in A$, a contradiction.

\section{REFERENCES}

1. V. Bergelson and N. Hindman, A combinatorially large cell of a partition of $N$, J. Combin. Theory Ser. A 48 (1988), 39-52.

2. __ Density versions of two generalizations of Shur's theorem, J. Combin. Theory Ser. A 48 (1988), 32-38.

3. __ Ultrafilters and multidimensional Ramsey theorems, Combinatorics 9 (1989), 1-7.

4. J. Berglund and K. Hoffman, Compact semitopological semigroups and weakly almost periodic functions, Lecture Notes in Math., vol. 42, Springer-Verlag, Berlin and New York, 1967. 
5. J. Berglund, H. Junghenn, and P. Milnes, Compact right topological semigroups and generalizations of almost periodicity, Lecture Notes in Math., vol. 663, Springer-Verlag, Berlin and New York, 1978.

6. W. Deuber, Partitionen und Lineare Gleichungssysteme, Math. Z. 133 (1973), 109-123.

7. R. Ellis, A semigroup associated with a transformation group, Trans. Amer. Math. Soc. 94 (1960), 272-281.

8. __ Lectures on topological dynamics, Benjamin, New York, 1969.

9. __ Locally compact transformation groups, Duke Math. J. 24 (1957), 119-125.

10. Z. Frolik, Sums of ultrafilters, Bull. Amer. Math. Soc. 73 (1967), 87-91.

11. H. Furstenberg, Recurrence in ergodic theory and combinatorial number theory, Princeton Univ. Press, Princeton, N. J., 1981.

12. H. Furstenberg and Y. Katznelson, Idempotence in compact semigroups and Ramsey theory, manuscript.

13. H. Furstenberg and B. Weiss, Topological dynamics and combinatorial number theory, J. Analyse Math. 34 (1978), 61-85.

14. L. Gillman and M. Jerison, Rings of continuous functions, Van Nostrand, Princeton, N. J., 1960.

15. R. Graham, B. Rothschild, and J. Spencer, Ramsey theory, Wiley, New York, 1980.

16. N. Hindman, Minimal ideals and cancellation in $\beta N$, Semigroup Forum 25 (1982), 291310.

17. __ Partitions and sums and products of integers, Trans. Amer. Math. Soc. 247 (1979), 227-245.

18. - The existence of certain ultrafilters on $N$ and a conjecture of Graham and Rothschild, Proc. Amer. Math. Soc. 36 (1972), 341-346.

19. _ The ideal structure of the space of $\kappa$-uniform ultrafilters on a discrete semigroup, Rocky Mountain J. Math. 16 (1986), 685-701.

20. __ Ultrafilters and combinatorial number theory, Lecture Notes in Math., vol. 751, Springer-Verlag, Berlin and New York, 1979, pp. 119-184.

21. __ Ultrafilters and Ramsey theory - an update, Proceedings of Set Theory and its Applications, Conference at York (to appear).

22. N. Hindman and J. Pym, Free groups and semigroups in $\beta N$, Semigroup Forum 30 (1984), 177-193.

23. A. Lisan, The ideal structure of the space of ultrafilters on a discrete semigroup and related Ramsey theorems, Dissertation, Howard University, 1988.

24. P. Milnes, Compactification of topological semigroups, J. Australian Math. Soc. 15 (1973), 488-503.

25. R. Rado, Note on combinatorial analysis, Proc. London Math. Soc. 48 (1943), 122-160.

26. W. Ruppert, Rechstopologische Halbgruppen, J. Reine Angew. Math. 261 (1973), 123-133.

Department of Mathematics, Howard University, Washington, D.C. 20059

Department of Mathematics, Ohio State University, Columbus, Ohio 43210 